\title{
Merging race models and adaptive networks: A parallel race network
}

\author{
DENIS COUSINEAU \\ Université de Montréal, Montréal, Québec, Canada
}

\begin{abstract}
This article presents a generalization of race models involving multiple channels. The major contribution of this article is the implementation of a learning rule that enables networks based on such a parallel race model to learn stimulus-response associations. This model is called a parallel race network. Surprisingly, with a two-layer architecture, a parallel race network learns the XOR problem without the benefit of hidden units. The model described here can be seen as a reduction-of-information system (Haider \& Frensch, 1996). An emergent property of this model is seriality: In some conditions, responses are performed with a fixed order, although the system is parallel. The mere existence of this supervised network demonstrates that networks can perform cognitive processes without the weighted sum metric that characterizes strength-based networks.
\end{abstract}

The objective of this article is to expand our knowledge of race models by showing the existing similarities between race models and connectionist networks. To this end, the architecture of race models is extended into the form of a network of connections, and a learning rule is introduced whose purpose is similar to the delta rule (Widrow \& Hoff, 1960). With such a common denominator, race models and connectionist models will, for the first time, be comparable. In this article, I introduce this integration of race and network approaches in the form of a parallel race network (PRN) model. One feature that is introduced in this network is redundancy of channels. Redundancy might be an important aspect of the human brain and, thus, should be considered when a network model of the brain is presented.

\section{Overview of the Single-Channel Race Models}

Accumulator models and random walk models are generalizations of the signal detection theory (SDT), sharing the same core assumption: One or many samples

Parts of this work were presented to the Conférences du Groupe de Recherche en Neuropsychologie, Université de Montréal, February 2001, to the Thirty-Third Annual Meeting of the Society for Mathematical Psychology, Kingston, August 2000, and to the Hoosier Mental Life, South Bend, March 2000. Sample programs can be found at http:// mapageweb.umontreal.ca/cousined/papers/07-prn/. The examples from the second section are solved by a deterministic (no learning) PRN at this location (the PRMod.m module). A program incorporating the learning rule is also available (the PRNet.m module). This research was supported by the Fonds pour la Formation de Chercheurs et l'Aide à la Recherche, Établissement de Nouveaux Chercheurs, and the Conseil pour la Recherche en Sciences Naturelles et en Génie du Canada. I thank M. Arguin, A. Criss, S. Hélie, G. L. Lacroix, C. Lefebvre, G. D. Logan, R. M. Nosofsky, D. Saumier, R. M. Shiffrin, J. T. Townsend, Trisha Van Zandt, and three anonymous reviewers for their useful comments on earlier versions of this article. I also thank D. Bamber, Y. Lacouture, P. Murray, and M. Wenger for suggesting applications for the race model. This article is dedicated to the memory of Philip L. Smith. Correspondence concerning this article should be addressed to D. Cousineau, Département de Psychologie, Université de Montréal, C. P. 6128, succ. Centre-ville, Montréal, QC, H3C 3J7 Canada (e-mail: denis.cousineau@umontreal.ca). are sequentially received from the senses (Green \& Swets, 1966). However, contrary to SDT, accumulator models and random walk models can take an arbitrary number of samples, this number changing from trial to trial. In these models, the samples can be evidence for one response or an alternative response (Townsend \& Ashby, 1983). In some variants, the samples can also be evidence for both responses or neither. Following Smith and Vickers (1988), the distinction between random walks and accumulator models lies in the nature of the evidence accumulation process. For the former, the accumulation process is not independent, because an evidence for one response implies a reduction of the amount of evidence for the alternative response. For the latter, the evidence accumulations are done on independent accumulators. However, the first accumulator filled triggers a response - thus, the name of race model.

The class of accumulator models can be further broken down by whether the evidence collected is discrete, called simple accumulator models by Luce (1986), or continuous, called strength accumulator models (Laberge, 1962). Examples of simple accumulator models are given by Audley and Pike (1965), where the sampling process takes a fixed amount of time, and by Pike (1973), where the time between two samples is continuous. An example of a strength accumulator is given by Smith and Vickers (1988), where the time between two samples is fixed. Another distinction between race models is whether the channels bringing evidence to the accumulator are dependent or independent (Meijers \& Eijkman, 1977).

All these models share one limitation: The evidence must travel sequentially through a single channel. As a result of this constrained architecture, the accumulators are located after a bottleneck. Yet, as has been noted by Thorpe and Gautrais (1999), there might not be enough time for information jam. These authors "recently demonstrated that the human visual system can process previously unseen natural images in under $150 \mathrm{~ms}$... To reach 
the temporal lobe in this time, information from the retina has to pass through roughly ten processing stages. If one takes into account the surprisingly slow conduction velocities of intracortical axons, it appears that the computation time within any cortical stage will be as little as $5 \mathrm{ms"} \mathrm{(p.} \mathrm{1).} \mathrm{Introducing} \mathrm{a} \mathrm{multiple-channel} \mathrm{archi-}$ tecture eliminates the bottleneck. This was one of the motivations for the model described in this article, since it is a fully parallel race model.

An alternative approach is the class of models known as the connectionist network models. Beyond the fact that they can predict choice data, they incorporate a learning mechanism. One utility of adding this mechanism to a network model is to predict the general pattern of learning. More important, it is used to reduce the number of free parameters. For example, consider a random walk model defined by boundaries and drift rates (e.g., Ratcliff, Van Zandt, \& McKoon, 1999; Ward \& McClelland, 1989). If these parameters could be learned by exposure to stimulus-response pairs, they would no longer be free to vary. The only free parameters remaining would correspond to a description of the random fluctuations in stimulus sampling (i.e., variability). ${ }^{1}$

In the early connectionist networks (Rosenblatt, 1961; Widrow \& Hoff, 1960), the inputs were assumed to reflect activation strength. The connections, loosely speaking, code the relevance of the input with respect to the output. The decision is based on a sum of the inputs' activations weighted by their relevance. The connections came to be labeled the weights. Beyond the weighted sum, some researchers added a threshold to the output. They were either soft, using a sigmoid function (Hinton, 1992; McClelland \& Rumelhart, 1988), or hard, with an absolute threshold (Arguin \& Bub, 1995; Page, 2000).

\section{Overview of the Article}

The general assumption in the connectionist models is that the relevant information takes the form of strength (strength of activation, strength of association). As such, a general label for those models is strength-based models. The strength-based networks, in conjunction with the weighted sum computations, definitely have had and continue to have a profound impact in many fields. Yet I believe that other approaches to cognition are possible. The one developed in this article, congruent with race models, assumes that time is critical for deciding which response to make. Furthermore, the connections are not described by how much evidence they bring but, rather, by when evidence is received. Thus, the model presented here is totally built around the notion of time and should be labeled, more generally, a time-based network.

In what follows, I will describe this extension to race models that I call the $P R N$. It is based on a fully connected network of inputs and outputs. Furthermore, this model has a learning rule, so that it can associate inputs with outputs through supervised learning. However, here ends the similarities between the time-based and the strength-based networks. The PRN is specifically aimed at predicting response times. Furthermore, this model can learn to solve nonlinearly separable problems (the XOR problem) without the benefit of hidden units.

\section{A DETERMINISTIC PARALLEL RACE NETWORK}

The purpose of this section is to introduce all the key elements of the PRN. The idea is to cast the race model into a network of connections identical to those used in classic (feed-forward) connectionist networks (see McClelland \& Rumelhart, 1988; Rosenblatt, 1961). The only differences are that PRN (1) does not consider the strength of activation and (2) does not use a weighted sum transmission rule. The notion of connection strength is totally irrelevant for PRN, since the inputs are assumed to be binary (activated or not). All it needs to know is when the input is activated. Thus, inputs are considered with respect to the time dimension. A present input activates its connections rapidly, as early as time $t=0$ if there is no randomness. On the other hand, an absent input will never activate its connections. Hence, a time $t=\infty$ can be assigned for an absence of input. This is a major change in input representation: In PRN, zero and infinity mean present and absent, respectively, whereas in strengthbased networks, one and zero have these meanings.

The output layer of PRN is composed of accumulator units. Their purpose is to get filled, at which time they fire. Each accumulator has a certain number of slots, represented by the accumulator size.

Between the inputs and the outputs are connections, also called, hereafter, channels. As is the case for the input, the single relevant property of the connections is represented in the time domain. Some connections can be very efficient and introduce almost no delay in the delivery of information. Other connections may be less efficient (e.g., impose a stronger resistance) to the transmission of information, so that delays will result. A connection with an infinite delay is simply a broken connection. Hence, connections with delays of zero are very effective channels whereas connections with infinite delays are off, congruent with the input representation.

Each of these concepts will be discussed in more detail in this section, along with two subsidiary aspects of the $\mathrm{PRN}$ - namely, redundancy and clock units. Concepts that are not covered in this section but will be later are (1) a fully elaborated mathematical notation, which will reveal important parallels between the PRN and strengthbased networks (see the "Mathematical Notations" section) and (2) the description of a learning rule. Assuming that the resistance to the transmission of information can be altered by exposure to stimulus-response pairs (supervised learning), a simple learning rule can be proposed that is described in the section "A PRN With a Learning Rule." The "Deterministic Solutions to Simple Problems" section presents examples of problems where the PRN can respond accurately, using only time as a source of evidence. 


\section{Description of the PRN}

The PRN assumes that information (or activation, essentially the same thing in the parallel race model) travels along channels with a finite velocity. As such, transmission times are the modus operandi of this model. What is crucial is the moment at which the inputs become activated and at which they reach the accumulators. I show here how a PRN can integrate confusable inputs into distinguishable patterns of response by manipulating these delays.

Input and output units. In its simplest form, a parallel race model is composed of inputs and outputs, as can be seen in Figure 1A. The purpose of the input units is to transmit the signals coming from the stimulus. Each output is an accumulator (an all-or-none or hard-threshold unit) with only integer value sizes. As was mentioned earlier, the inputs are not described by how strong the signal is but by when the signal is available. In this article, it is assumed that a trial begins at time $t=0$, although this choice is arbitrary.

Architecture. As is generally the case in strengthbased networks, all the inputs are connected to all the outputs. All these channels transmit information (often termed evidence in the following). In PRN, the channels are described by how well the information flows through them. A very efficient channel will transmit the information with but a minimal delay. It is assumed arbitrarily that the smallest delay can be zero, representing highly efficient channels. At the other extreme, an off connection will be labeled with an infinite delay.

The delays are neither arbitrary nor constant. They are set specifically to solve a problem - that is, to answer to the stimulus with the correct response in a given context. For example, if an evidence is highly diagnostic for a response, it should reach the relevant accumulator with no delay. One purpose of the learning rule described in the section "A PRN With a Learning Rule" is to change the delays following exposures to the stimulus-response pairs.

Redundancy. Because it is unlikely in a large system that information travels along only one path, I introduce redundancy in the inputs. Redundancy is obtained by simply duplicating each input by a factor $\rho$; hence, the total number of inputs is actually multiplied by $\rho$. Redundancy is not a necessary ingredient of the PRN. This model can respond accurately to input in most problems without redundant input (one exception is the XOR problem, which is discussed in the Simulations section).

Thresholds and decision. In the PRN, each output is an accumulator with $K$ slots. An accumulator makes a response when all its slots are filled. This happens when the $K$ th fastest input reaches the accumulator. All the accumulators can differ on their threshold size $K$. A second purpose of the learning rule is to adjust the size of the accumulators when exposed to stimulus-response pairs, so as to collect just the right amount of evidence. When an output is triggered, it simply starts firing. However, all the accumulators are in competition: Only the one filled first fires and ends the trial. This is the reason for the term race models.

Clocks. Discrimination is difficult when the system has to make an answer for nothing presented (an accumulator responding to zero evidence is meaningless). Hence, the system must be able to decide that after a certain amount of time, if the other accumulators have not reacted, it is time for a nothing-presented response. This implies that time is a source of evidence in itself.

Perception of time can be achieved by the presence of clocks whose sole purpose is to tick at regular intervals. There exists empirical evidence for the existence of such clocks in brains. Oscillatory circuits (sometimes called pacemakers) have been identified in the nervous system of many animals, including mammals (Changeux, 1983;
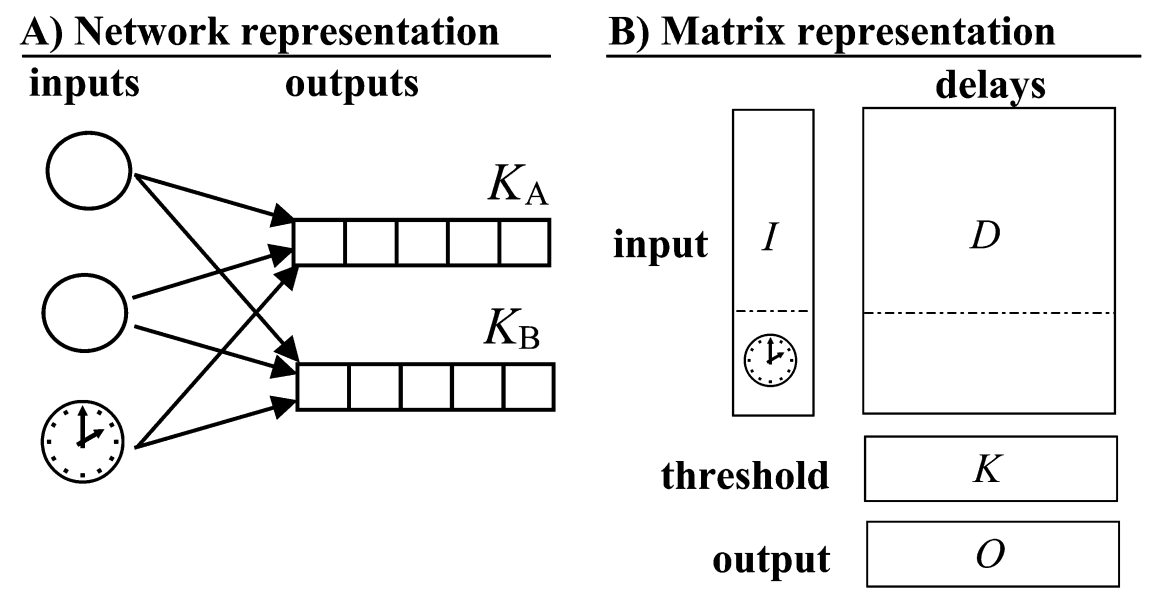

Figure 1. A parallel race model with two accumulators and one clock unit. (A) Signals on input $i$ are received at various moments $I_{i}$ and stored in the accumulator $j$ after going through the connections with some delay $D_{i j}$. The symbol $\stackrel{\perp}{\circ}$ denotes the clock unit. (B) The same model seen as a matrix of delays and vectors for inputs, thresholds, and outputs. The dashed line indicates the delimitation between inputs from the stimulus and inputs from the clock units. 
Chaplain, 1979; Levitan, Harmar, \& Adams, 1979). Furthermore, human behavioral data in favor of internal clocks were reported by Rousseau and Rousseau (1996) and Hopkins and Kristofferson (1980). In the last study, subjects had to perform a tempo completion task (listen to two taps and press a button when the third tap should occur). The results are exceptional (standard deviation in response times of $7 \mathrm{msec}$ and symmetrical distributions), and they strongly support the existence of "internally timed delays which can be inserted in the S-R chain" of processing (p. 241).

Thus, because some decisions are default responses when the network perceives nothing, extra temporal inputs are needed. I call them clock units, denoted with the symbol $(1)$. Clocks do not have a special status in the PRN; they are extra inputs, in addition to those related to the stimulus. However, the activity of the clocks does not depend on the actual pattern of input presented. They are always on. Therefore, the clock units are activated on every trial. The number of clocks is arbitrary (and can be large), but in many situations, one clock is sufficient. Figure 1 shows a PRN with one clock.

These are all the ingredients of a PRN, and with adequately set delays in the connections and adequate accumulator sizes, it can respond accurately to many inputs with the appropriate outputs. In the next section, I will illustrate how a PRN can be configured to solve a set of simple input-output problems.

\section{DETERMINISTIC SOLUTIONS TO SIMPLE PROBLEMS}

The purpose of this section is to illustrate, with four example problems, how the PRN can make correct outputs to given inputs. Although the PRN is relatively simple, it is our experience that people acquainted with strength-based networks have difficulties understanding it because some of the basic concepts, although similar looking, have in fact the opposite meanings. The problems are extremely simple. They were chosen so that they can be simulated with paper and pencil. The four problems are the detection problem, the one-dimensional problem, the identification problem, and the and problem. Figure 2, left column, illustrates the problem spaces. The dimensions represent the values of certain attributes of the stimuli. Here, the attributes are all binary, which means present or absent.

When working with binary features, it is convenient to think in terms of zeros and ones, and indeed, the problem spaces in Figure 2 are organized in that way. However, when presenting a stimulus to the network, what is crucial is whether the information on the dimension has arrived (i.e., is present) or has not arrived yet (i.e., is not present). As such, activated units receive an input right away (at time $t=0$ ), whereas inactivated units never receive an input (i.e., they will be activated after an infinite amount of time, $t=\infty)$. This distinction is very important because what is fed into the network are the times at which the activations occur, not their strengths. Therefore, instead of thinking in terms of 0 and 1 (absent or present), one should think in terms of $\infty$ and 0 (never or immediate). It is assumed that at the end of a trial, all the accumulators are reset at zero.

All the toy problems presented here require at least one clock unit to deal with the nothing-presented case. For simplicity's sake, it is assumed that these clock units are always immediately on, so that their activation times are all zero.

For the present illustrations, I deliberately ignored variability in the moments of activation. It is then an exercise in logic to find the optimal solutions. The learning rule is also not considered in this section. A Mathematica program that simulates a deterministic PRN is available on the author's Web site (the PRMod.m module, http:// mapageweb.umontreal.ca/cousined/papers/07-PRN), along with a document where the following examples are solved.

\section{Example 1: The Detection Problem}

A simple problem is what I call the detection problem. This problem is composed of one input $\left(I_{1}\right)$, and the task of the system is to respond $\mathrm{A}$ if this dimension is on (present right away, $I_{1}=0$ ) and to respond $\mathrm{B}$ if the dimension is off (never presented, $I_{1}=\infty$ ). Figure $2 \mathrm{~A}$, on the left, shows the problem space.

In terms of connections, if the input gets activated, it should be conveyed to the output A with no delays. This connection should be immediate and, thus, receive a value of 0 (or the smallest possible delay on such a channel). In addition, this single piece of information is sufficient for an A response, so the size of Accumulator A must be 1 . Because this same information is totally irrelevant to Response $\mathrm{B}$, it should never reach the $\mathrm{B}$ accumulator, so there should be no connection between the input and the B response. Instead, Accumulator B should rely on a clock unit. However, the activation of the clock should not reach the B accumulator immediately. If such were the case, in the presence of a signal on the input, both Accumulators A and B would be triggered at the same time, and the tie would make the response ambiguous. The solution is to delay the clock signal a little, in case an input signal is present. Thus, there should be a delay $t>0$ between the clock signal and Response B.

Figure $2 \mathrm{~A}$, on the right, summarizes the solution, using a matrix of connection delays and a vector of accumulator sizes. The number $D_{i j}$ at the intersection of the $i$ th line and the $j$ th column in the $D$ matrix is the connection delay between the $i$ th input unit $(1,2$, or $(2)$ for the clock unit) and the $j$ th output unit (Accumulator A or $\mathrm{B}$ in this example). Thus, $D_{\odot \mathrm{A}}$ is the delay in the connection between the clock and the first accumulator. Numbers below the dashed lines are connected to clock units. Furthermore, let $K_{j}$ represent the size of the $j$ th accumulator. The $j$ th number in the $K$ vector is the accumulator size of the $j$ th output unit.

\section{Example 2: The One-Dimensional Problem}

In this problem, inputs are composed of two dimensions $\left(I_{1}\right.$ and $\left.I_{2}\right)$, with each dimension taking on the values present or absent $(0$ or $\infty)$. The correct response is $\mathrm{A}$ 
A) Detection problem

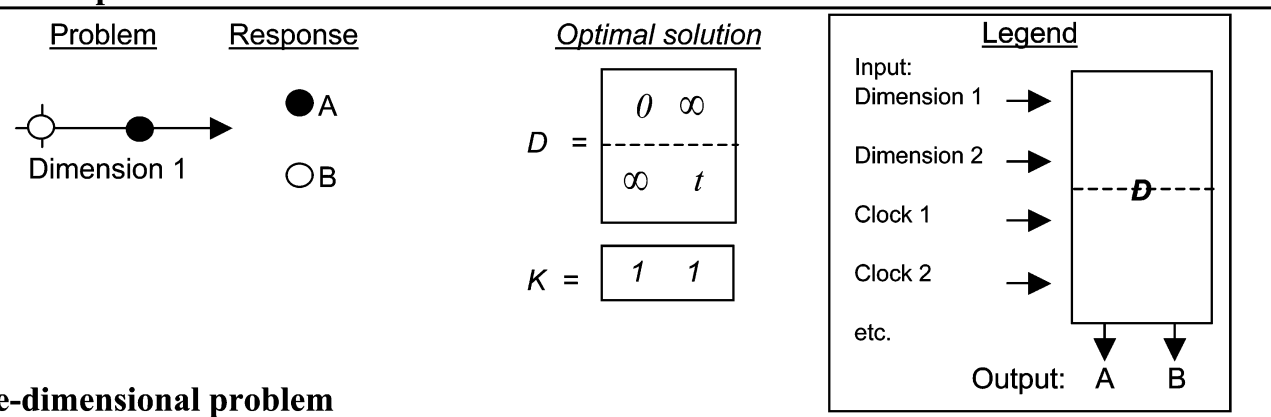

B) One-dimensional problem

\section{Optimal solution}

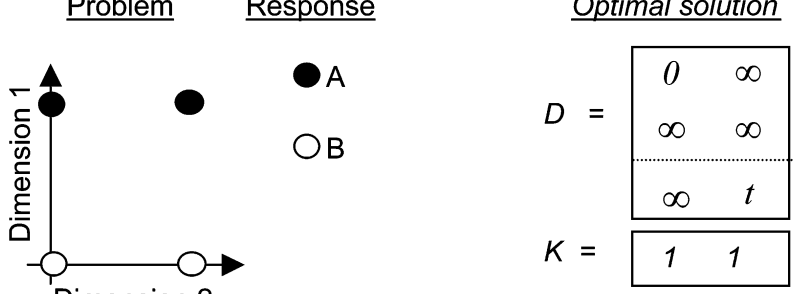

\section{C) Identification problem}

\begin{tabular}{|c|c|c|c|c|c|}
\hline Problem & Response & \multicolumn{4}{|c|}{ Optimal solution } \\
\hline & A & & & $\infty$ & $\infty$ \\
\hline & OB & $D$ & $\infty$ & 0 & $\infty$ \\
\hline & $\square \mathrm{C}$ & & $\infty$ & $\infty$ & $t$ \\
\hline Dimension & & $K=$ & 1 & 1 & 1 \\
\hline
\end{tabular}

D) And problem

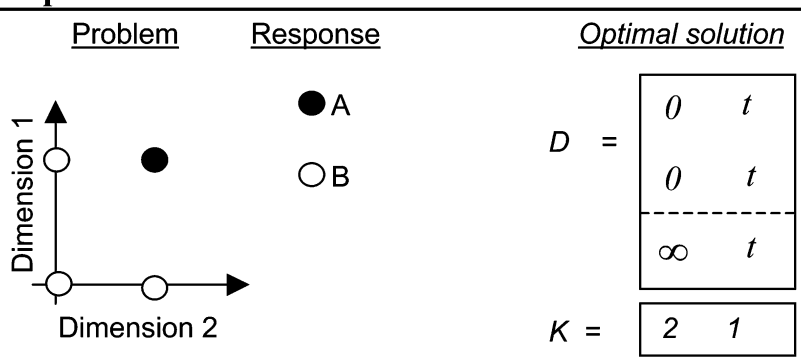

Figure 2. The problems in the "Deterministic Solutions to Simple Problems" section. The left column shows the problem space with the associated responses. Note that a value of zero on any dimension indicates that the corresponding feature was absent. The right column shows the matrix solution for a parallel race network. (A) The detect problem. The system has to say " $A$ " if something happens. The general solution is to respond " $A$ " as soon as Dimension 1 is activated $\left(D_{1 \mathrm{~A}}=0\right)$ or else respond "B" after a while $\left(D_{{ }_{B}}=t>0\right)$. (B) The one-dimensional problem. The correct response is " $A$ " if and only if Dimension 1 is presented. Input for Dimension 1 has the highest priority for Response $A\left(D_{1 \mathrm{~A}}=0\right)$, followed by input from the clock with respect to $\operatorname{Response} B\left(D_{\odot B}=t>0\right)$. Thresholds are 1. (C) The identification problem. Correct responses are "A" or "B," depending on which dimension is on. There is no case in which both dimensions are on at the same time. The clock is used for a nothing-presented Response $C$ (at time $D_{C}=t$ ). (D) The and problem and its solution. The correct response is $A$ if and only if the two dimensions are presented. Input from both dimensions have the highest priority $\left(D_{1 \mathrm{~A}}=D_{2 \mathrm{~A}}=\right.$ $0)$, but both are needed $\left(K_{\mathrm{A}}=2\right)$. Any input or clock can later provoke Response $\mathrm{B}\left(D_{i \mathrm{~B}}=t>0\right)$. The clock is essential in case no input is presented. 
if Dimension 1 only is present. This problem is called a one-dimensional (1-D) problem because only one dimension is relevant. The presence or absence of the other dimension is totally uninformative. Figure $2 \mathrm{~B}$, on the left, shows the problem space.

Because the first input is fully diagnostic of Response A, it should be conveyed immediately to Accumulator A, and a response can occur immediately-thus, $D_{1 \mathrm{~A}}=0$. In addition, this information is sufficient, so that the accumulator size for Response $\mathrm{A}$ is $1\left(K_{\mathrm{A}}=1\right)$. The network should answer Response $B$ if and only if the first input is not stimulated. But in that case, $I_{1}=\infty$, and the network would wait indefinitely. Thus, the network, at some time, must commit itself and respond B. The clock unit is used for that purpose: Let $D_{\oplus \mathrm{B}}=t>0$ be the delay before the clock activation reaches Accumulator B. The delay $t$ must be significantly larger than 0 , or else Response A and Response B would occur approximately at the same moment. The right column of Figure 2B shows the solution in terms of delays and thresholds.

The solution shows that Response B will be performed if and only if Response A was not performed previously. In a certain sense, Response B must agree not to compete with Response A in cases in which the input on Dimension 1 is activated. This form of collaboration is in direct opposition to strength-based networks, where the inputs are in competition.

\section{Example 3: The Identification Problem}

The identification problem is an example where the system must answer A if Dimension 1 is on and B if Dimension 2 is on (the case in which both are on is assumed to be nonexistent). When neither Dimension 1 nor Dimension 2 is present, the system must make a third response, called C. Figure $2 \mathrm{C}$, on the left, shows the problem space.

Apart from a third accumulator, this example is simple to solve. As soon as something is perceived on Dimension 1 or 2, Response A or B, respectively, must be made. Thus, the delays $D_{1 \mathrm{~A}}$ and $D_{2 \mathrm{~B}}$ are set to 0 . However, the clock is responsible for making Response $\mathrm{C}$, and to avoid ambiguity with $\mathrm{A}$ and $\mathrm{B}$, it should be delayed: $D_{\odot \mathrm{C}}=t>0$. Figure $2 \mathrm{C}$, on the right, shows the solution matrix.

\section{Example 4: The And Problem}

The and problem requires that both inputs be present for the system to respond A. All other patterns of input must result in Response B. Figure 2D shows the problem space.

The correct solution requires that the inputs be transmitted rapidly to Accumulator A. However, the two must be present. This requires that the threshold $K_{\mathrm{A}}$ be 2 . If the conjunction is not present, any single input will not fill Accumulator A. However, a single input will trigger a B response after some time, to avoid a tie. A clock unit can also trigger a $\mathrm{B}$ response $\left(D_{\odot \mathrm{B}}=t>0\right)$. Figure $2 \mathrm{D}$, on the right, shows the solution matrix.

Because Response B is the same whether there is only one input or none, the clock can be used by itself to respond $\mathrm{B}$, and the other two connections can be removed
( $D_{1 \mathrm{~B}}$ and $D_{2 \mathrm{~B}}$ to $\infty$ ), without any change in performance or accuracy.

\section{MATHEMATICAL NOTATIONS}

The PRN can be represented by drawing input units connected to accumulators (as in Figure 1A). However, a vectorand-matrix representation is more convenient, both for its compactness and to compute the output of the network. Such an approach is widely used when strength-based networks are studied. For example, in a two-layer network, the input and the connection weights are often represented by a vector $I$ and a matrix $W$. The output is then given by a standard inner product $O=I . W$ (the dot is used to show explicitly the presence of an inner product), which performs a weighted sum of the input. However, the notion of race in the PRN does complicate things: The standard inner product would return the average time, not find the fastest accumulator. In the following, I will propose a simple modification to the inner product that overcomes this problem by introducing minima into vector operations.

\section{Representation of the Inputs and the Connections}

Let us assume that the input is composed of $n_{I}$ dimensions. Suppose further that there are $n_{\odot}$ clock units. A vector $I$ can be defined that contains all the information from the input and the clocks. Formally, let $I_{i} \geq 0$ denote the moment when the $i$ th input gets activated, and let $I$ denote the input vector $\left\{I_{i}\right\} . I$ is of size $\rho \times\left(n_{I}+n_{\odot}\right)$, where $\rho$ is the redundancy factor. Because the clocks are always on, one can view $I$ as the input vector augmented by the time at which the clocks turn on.

All the inputs and the clocks have connections to the accumulators, represented by $D_{i j} \geq 0$, the delay between the activation of the input $i$ and the moment at which one slot of accumulator $j$ is filled. Informally, the $D_{i j}$ can be seen as conduction times. In order to connect all the inputs $i$ to all the outputs $j$, let the delay matrix $D$ be a twodimensional matrix $\left\{D_{i j}\right\}$. To avoid confusion, the accumulators are labeled using uppercase letters, so that $j$ can take the values A, B, C, and so on. $D$ can also be seen as a matrix of connections for the input, augmented by a matrix of connections for the clocks.

There is no restriction on the connections. Some input may bring evidence in favor of one response, some to the other, and some to both. There may even be input that will contribute to no accumulator. For example, if $D_{i \mathrm{~A}}$ is zero, this means that as soon as input $i$ is activated, this activation immediately fills one slot of Accumulator A (no delay). If, on the other hand, $D_{i \mathrm{~A}}$ is infinite, this piece of evidence will never reach the accumulator. In other words, input $i$ is not an evidence in favor of Response A. Finally, if $D_{i \mathrm{~A}}=D_{i \mathrm{~B}}$, input $i$ is equally important to decide whether the response is A or B (with respect to Response $\mathrm{C}$, for example).

Let $K_{\mathrm{A}}$ denote the size of the first accumulator associated with Response A, and in general, $K_{j}$ the size of the 
$j$ th accumulator. Further, let the vector $K$ denote all the accumulator sizes $\left\{K_{j}\right\}$.

Figure 1B illustrates the three components, using boxes: $I$ (changing with the input) and $D$ and $K$ (describing the network).

For modeling purposes, the time at which the clocks become active $\left(I_{\odot}\right)$ has to be specified. In the following examples, I set this time to 0 : The clocks are immediately active. This is unrealistic, but it is not important, since delays in connections $D_{\odot j}>0$ can compensate for fast clocks, delaying this temporal evidence.

\section{Redefining the Inner Product}

The response made by the PRN is determined by which accumulator responds first. The time at which inputs become accessible $\left(I_{i}\right)$ and the delays in connections $\left(D_{i j}\right)$ are additive. Thus, a combination of sum and minima is required. It turns out that a simple modification to the inner product accommodates the needs of the race model. Furthermore, adopting this redefined inner product yields a very compact notation that allows convenient comparisons with strength-based networks that use the standard inner product in their transmission rule.

The standard inner product is defined on onedimensional vectors $U$ and $V$ by

$$
U . V:=\sum_{i=1}^{n}\left(U_{i} \times V_{i}\right)
$$

where $n$ is the size of both vectors. One can note in the definition above that there are two operators at work: a joining operator $(X)$ and an aggregate operator $(\Sigma)$. The role of the joining operator is to relate a couple $\left\{U_{i}, V_{i}\right\}$, defined in a domain $\mathbb{R}^{2}$ (the real domain is assumed in this article) with a single value in the $\mathbb{R}$ domain. The multiplication $\times$ does constitute an appropriate joining operator in standard situations. Having joined all the couples from the two vectors, one obtains a list of values. This list needs to be reduced to a summary value, and that is the role of the aggregate function (defined on the domain $\mathbb{R}^{n} \rightarrow \mathbb{R}$ ). Again, the summation $\Sigma$ satisfies this requirement. To avoid confusion, a more explicit notation

$$
U\left(\begin{array}{l}
x \\
\Sigma
\end{array}\right)^{V}
$$

can be used to represent the standard inner product $U . V$, based on the multiplication for the join operator and the summation for the aggregate operator.

The redefined inner product that is introduced for race models can similarly be denoted

$$
U\left(\begin{array}{c}
+ \\
\text { Min }
\end{array}\right)^{V}
$$

where Min is the minimum operator. The idea is that the multiplication is changed by an addition, and the summation by a minimum. It is thus defined by

$$
U\left(\begin{array}{c}
+ \\
\text { Min }
\end{array}\right) \quad V:=\operatorname{Min}_{i=1}^{n}\left(U_{i}+V_{i}\right) \text {. }
$$

Here is an example. Let $U=\left(\begin{array}{lll}1 & 8 & 1\end{array}\right)$ and

$$
V=\left(\begin{array}{l}
2 \\
8 \\
5
\end{array}\right)
$$

be two vectors. First, perform the joining operation, using an addition:

$$
U+V=\left(\begin{array}{l}
1 \\
8 \\
8
\end{array}\right)+\left(\begin{array}{l}
2 \\
8 \\
5
\end{array}\right)=\left(\begin{array}{c}
3 \\
16 \\
6
\end{array}\right) .
$$

Second, follow with an aggregation done by locating the smallest of the list:

$$
\operatorname{Min}\left(\begin{array}{c}
3 \\
16 \\
6
\end{array}\right)=3
$$

On some occasions, it is not the first minima that is required, but the second or third, and so on. Let us define the $K$ th minimum operator $\mathrm{Min}_{K}$ as a function that returns the $K$ th smallest value of a vector. In the above example,

$$
U\left(\begin{array}{c}
+ \\
\operatorname{Min}_{2}
\end{array}\right)^{V}
$$

would yield 6 .

The redefined inner product can easily be extended to accommodate matrices. In addition, if $K$ is a vector containing $m$ accumulator sizes, instead of a scalar, and $A$ likewise has $m$ columns, the redefined inner product of a vector and a matrix is defined by

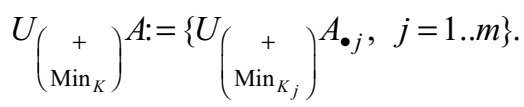

That is, for the $j$ th column of $A$, we perform an inner product with $U$ (as defined in Equation 1), using the $K_{J}$ th accumulator size.

As an example, given

$$
U=\left(\begin{array}{lll}
1 & 4 & 3
\end{array}\right), A=\left(\begin{array}{ll}
2 & 1 \\
5 & 4 \\
7 & 3
\end{array}\right) \text {, and } K=\left(\begin{array}{ll}
1 & 2
\end{array}\right),
$$

the result of

$$
U_{\left(\begin{array}{c}
+ \\
\operatorname{Min}_{K}
\end{array}\right)}^{A}
$$

is given by first applying the joining operation columnwise:

$$
U+A=\left(\begin{array}{cc}
3 & 2 \\
9 & 8 \\
10 & 6
\end{array}\right)
$$


then aggregating by finding the $K_{j}$ th minimum on the $j$ th column:

$$
\begin{aligned}
\operatorname{Min}_{K}\left(\begin{array}{cc}
3 & 2 \\
9 & 8 \\
10 & 6
\end{array}\right) & =\left[\operatorname{Min}_{K 1}\left(\begin{array}{c}
3 \\
9 \\
10
\end{array}\right), \operatorname{Min}_{K 2}\left(\begin{array}{l}
2 \\
8 \\
6
\end{array}\right)\right] \\
& =\left[\operatorname{Min}_{1}\left(\begin{array}{c}
3 \\
9 \\
10
\end{array}\right), \operatorname{Min}_{2}\left(\begin{array}{l}
2 \\
8 \\
6
\end{array}\right)\right] \\
& =\left(\begin{array}{ll}
3 & 6
\end{array}\right) .
\end{aligned}
$$

When the context is not misleading, a more compact notation $U \sim A$ will be used to stand for the redefined inner product

$$
U_{\left(\begin{array}{c}
+ \\
\operatorname{Min}_{K}
\end{array}\right)}^{A .}
$$

With the compact notation, however, it is not explicit that the vector $K$ is involved.

\section{Transmission Rule}

Let us go back to the PRN. The time for the $i$ th input to reach the $j$ th accumulator is given by the sum $I_{i}+D_{i j}$. Thus, the first slot of accumulator $j$ will be occupied by the fastest of all the $\left\{I_{i}+D_{i j}\right\}$, at a time given by $\operatorname{Min}\left\{I_{i}+D_{i j}\right\}$. The second slot will be filled when the second fastest input reaches the accumulator, and so on, until $K_{j}$ slots are filled. The output of this accumulator occurs at time $O_{j}$ when the $K_{j}$ th fastest input reaches the accumulator.

To summarize these computations, the standard inner product cannot be used, because it is a combination of product and summation and the PRN requires that the fastest time be kept, not the average time. However, the redefined inner product $\sim$ is appropriate.

With that operation, the response times for all accumulators given a pattern of input $I$ are contained in a vector $O=\left\{O_{j}\right\}$, given by

$$
O=I \sim D .
$$

However, only one response can be made, given by the first filled accumulator $j$, at time $t=\operatorname{Min}\left\{O_{j}\right\}$, the other responses being too late.

As an example, suppose the following PRN, having two inputs and one clock (no redundancy, $\rho=1$ ), defined by

$$
D=\left(\begin{array}{ll}
4 & 5 \\
1 & 3 \\
7 & 5
\end{array}\right), K=\left(\begin{array}{ll}
2 & 1
\end{array}\right) \text {. }
$$

When presented with the input $I=\left(\begin{array}{lll}0 & 5 & 0\end{array}\right)$, Accumulator A (whose connections are located in the first column of $D$ ) will have its first slot filled at time 4 (by the first input) and the second slot at time 6 (by the second input, arriving at 5 , but delayed by 1 in the matrix $D$ ).
Accumulator B (second column of $D$ ) will be full at time 5 (by the first input or the clock). Equivalently,

$$
I \sim D=\left(\begin{array}{lll}
0 & 5 & 0
\end{array}\right) \sim\left(\begin{array}{ll}
4 & 5 \\
1 & 3 \\
7 & 5
\end{array}\right)=\left(\begin{array}{ll}
6 & 5
\end{array}\right) .
$$

Because the first accumulator to fire is Accumulator B at time $t=5$, the trial ends at that moment with a $\mathrm{B}$ response.

Despite its awkward look, remember that $\sim$ is simply a (redefined) inner product. Whereas the standard inner product is at the core of strength-based networks, the redefined operation is at the core of time-based models.

\section{A PRN WITH A LEARNING RULE}

So far, the PRN turns out to be a flexible model, and a compact mathematical notation stems from it. However, the PRN cannot describe the dynamic of changes in $D$ and $K$ as learning occurs. This is in opposition to the strengthbased network models (McClelland \& Rumelhart, 1988), where the learning mechanism is fully described. This section will fill this gap by providing a learning rule for the PRN. The objective is that the network finds itself the right delays $D$ and the appropriate accumulator sizes $K$. The learning rule described is simple, and when I tested the resulting network with the problems described in the Deterministic Solutions to Simple Problems section, it always converged toward the optimal solutions. Overall, the learning rule described here tries to penalize the accumulator that reacted too rapidly by slowing down the channels that brought information to it. Mathematica source code for performing simulations of the network and its learning rule is also available on the author's Web site (the PRNet.m module).

\section{Description of the Learning Rule}

The physical implementation of the network stays the same as before (refer to Figure 1). As usual, inputs are considered to be signals coming from the stimulus. Because signals take time to travel, inputs are defined by the moment at which their activation reaches the input unit, not by their strength: Either a signal has arrived, or it has not yet arrived. As before, the connections $D$ between the inputs and the accumulators are not weights but delays in the transfer of information. Accumulators are filled by incoming signals; each signal takes one slot. Once the trial has ended, all the accumulators are emptied prior to a new trial.

Response times are computed in the same way as before, with one modification. As will be seen next, the learning rule allows thresholds $K$ to increase by noninteger values. Because only integers can be used for the accumulator sizes, the network uses the integer parts of the thresholds (denoted $\lfloor K\rfloor$ ) in the decision rule, which, in 
the more elaborated notation of the previous section, is denoted

$$
O=I\left(\begin{array}{c}
+ \\
\operatorname{Min}_{\lfloor K\rfloor}
\end{array}\right) D .
$$

As usual, only the fastest accumulator makes a response $j$ at time $O_{j}$ such that $O_{j}=\operatorname{Min}\{O\}$.

Because the learning rule is supervised, it is assumed that a "teacher" is present that indicates the correct response (i.e., which output unit should win the race). The response of the network can be either correct or erroneous. When it is erroneous, the output unit that fired is not the one indicated by the teacher. The first is said to have produced a false alarm (it has fired too soon while it should have remained silent), whereas the second missed (it remained silent while it should have won the race). Learning in the parallel race network takes place when an error occurs. There are two rules, one that updates the delays, and the other that updates the thresholds: (1) For the accumulator $j$, which produced a false alarm, one of the delays $D_{i j}$ responsible for that error (i.e., one of the $K_{j}$ th fastest delays $\left\{I_{i}+D_{i j}\right\}$ ) is increased by an arbitrary value $\varphi$. In other words, because that accumulator was filled too rapidly, one of its connections is slowed down to avoid this error again. (2) For the accumulator $j$ that missed, the threshold $K_{j}$ is modified. If the number of inputs that are active when the error is detected is larger than the value of $K_{j}$, the size of that accumulator is increased by an arbitrary value $\varepsilon$. On the other hand, if the number of active inputs is smaller than $K_{j}, K_{j}$ is decreased by $\varepsilon$. As a result, as long as errors occur, the accumulator size varies to reflect the number of active inputs. Note that contrary to intuition, increasing the accumulator sizes does not necessarily slow the responses, since information travels on parallel channels.

At the beginning of training, all thresholds $K_{j}$ are set to 1 , and delays $D_{i j}$ are set to random uniform numbers. Random initial delays are required to avoid tied decisions, because ties make the error attribution difficult to assess. The choices for $\varepsilon$ and $\varphi$ are arbitrary, and different values were tested with no qualitative change in the learning behavior of the network. The only restriction is that $\varepsilon$ be smaller than or equal to 1 ; otherwise, $\left\lfloor K_{j}\right\rfloor$ might not assume all the successive integers. For example, with $\varepsilon=1.5$, on the second miss of a given accumulator, the effective threshold would jump from $2(\lfloor 2.5\rfloor)$ to 4 $(\lfloor 4\rfloor)$, never testing the accumulator size 3 .

The learning rule above proved efficient on all the problems tested so far and resulted in learning that was surprisingly fast (see the next section). Yet it is not based on a priori principles (such as entropy reduction; Hinton, 1992) and, thus, might seem unprincipled. However, I believe it is a question of time before the justification of the learning rule above is found. First, the learning rule differs from the classic learning rules (such as the $\Delta$ rule and the Hebbian rule). Yet, recent work (Cousineau, Lacroix, \& Hélie, 2003) seems to indicate that the first rule above is very close in spirit to a $\Delta$ rule and can be rewritten to highlight the similarities between them. Second, it is not known whether the PRN performs a gradient descent search for the optimal solution, as do Perceptrons (Hinton, 1992; Kohonen, 1984). The difficulty lies in the redefined inner product, which is a new operator. Informal discussions with machine-learning researchers suggest that the PRN might be implementing a Lagrangian descent algorithm (LeCun \& Bottou, 2003). At this moment, it is not possible to derive a proof of convergence toward the optimal solution, contrary to many strengthbased networks, where such a proof exists. ${ }^{2}$

\section{Application to an Example}

To illustrate the learning rule, I will briefly describe the first two trials of a PRN trained with the and problem presented in the Deterministic Solutions to Simple Problems section. At first, the delay matrix is random, and the accumulator sizes are at 1 . Suppose that the matrix $D$ is given by

$$
D=\begin{array}{ccc} 
& \multicolumn{1}{c}{\mathrm{A}} & \mathrm{B} \\
\cline { 2 - 3 } I_{1} & 0.1 & 0.2 \\
I_{2} & 0.3 & 0.4 \\
\hdashline I_{\odot} & 0.5 & 0.6 \\
\cline { 2 - 3 } & &
\end{array}
$$

Comparing this matrix with the theoretical solution in Figure 2D, we see that many delays are inappropriate. For example, the delay between the clock and the first response is currently 0.5 (in arbitrary units of time), whereas it should be very large, since the clock is never involved in a conjunction-present response.

On the first trial, suppose the input has only Dimension 1 presented (not a conjunction). Adding the clock, the input is given by $I=(0 \infty 0)$. Computing $O$ yields $(0.10 .2)$. The first accumulator is the fastest and answers at time 0.1 , which is an error. Thus, Accumulator A false alarmed, whereas Accumulator B missed. According to Rule 1, the delay involved in the error is increased by $\varphi$ (say, 0.5). This is the connection $D_{1 \mathrm{~A}}$. Rule 2 does not come into play, since both the accumulator size for Response $\mathrm{B}$ and the number of inputs is 1 .

On the second trial, suppose that the conjunction is presented. Adding the clock, the input is $I=\left(\begin{array}{lll}0 & 0 & 0\end{array}\right)$. Computing $O$ yields ( 0.30 .2 ), resulting in a false alarm made by Accumulator B. From Rule 1, the delay 0.2 is increased by a quantity $\varphi$. From Rule 2 , the accumulator size of Response A is increased by a quantity $\varepsilon$ (say, 0.2 ), since it is currently of size 1 and the number of inputs presented (two) exceeds this value. After two trials, the system is described by

$$
\begin{array}{c|cc|} 
& \multicolumn{1}{c}{\mathrm{A}} & \mathrm{B} \\
\cline { 2 - 3 } I_{1} & 0.6 & 0.7 \\
I_{2} & 0.3 & 0.4 \\
\hline I_{\odot} & 0.5 & 0.6 \\
K= & (1.2 & 1.0)
\end{array} .
$$

By continuing this simulation, the first accumulator will rapidly stabilize at a size of 2 . As soon as the accumula- 
tors settle in a stable position, the shorter delays will increase as long as they create false alarms.

\section{SIMULATIONS}

The previous section presented the learning rule for the PRN. Application of this rule to deterministic input (no noise) is straightforward, and the reader is invited to simulate it to the problems presented in the Deterministic Solutions to Simple Problems section or check the Web site for the results. However, there is no certainty that this learning rule can learn and settle into a stable configuration of delays and thresholds when the inputs are noisy, redundant, or both.

In this section, I will explore the capabilities of the PRN to learn a difficult problem, the XOR problem. The XOR problem is a complex problem with an important history. Indeed, Minsky and Papert (1969) showed that the XOR problem is a linearly nonseparable problem (there exists no single linear boundary between the As and the Bs in the problem space) and cannot be solved by two-layered connectionist networks. As this example will show, however, a hidden layer is not necessary within the PRN architecture.

\section{Overview of the Simulations}

The XOR rule is to respond $\mathrm{A}$ if both or none of the inputs is present and $\mathrm{B}$ otherwise. Figure $3 \mathrm{~A}$ shows the prob- lem space in the usual format. Figure 3B illustrates the theoretical solution to the XOR problem, using the PRN.

When there is no redundancy $(\rho=1)$, the correct solution is to react rapidly if two inputs are present $\left(D_{1 \mathrm{~A}}\right.$ and $D_{2 \mathrm{~A}}$ equal $\boldsymbol{t}_{0} \approx 0$ ) and to be cautious if there seems to be no input (delays $\boldsymbol{t}_{2}$ for clock units). However, when only one input is present, Accumulator B should react more quickly than the clocks and more slowly than Accumulator A, to avoid simultaneous responses. So the delays between the inputs and Accumulator B should be set at $\boldsymbol{t}_{1}$, where $\boldsymbol{t}_{0}<\boldsymbol{t}_{1}<\boldsymbol{t}_{2}$. The threshold for an A response is 2 , since a conjunction is required. As a consequence, to make an A response when nothing is present, two activations are required. This means that two clock units are required. Note that the additional clocks do not change the problem space to a linearly separable one, since the clocks are always on. The threshold for Response B should be 1 , since only one evidence is available to fill Accumulator B. Because the clocks play no role with respect to Response B, the connections should be off or, at least, represent very long delays ( $\boldsymbol{t}_{3}$ large).

It seems as if the network could order the responses serially. However, this is not exactly true. The network operates in parallel, but absent information is given more time to arrive.

When redundancy is present ( $\rho>1)$, Accumulator A's receiving two inputs does not provide definitive evidence

\section{A) Problem space}

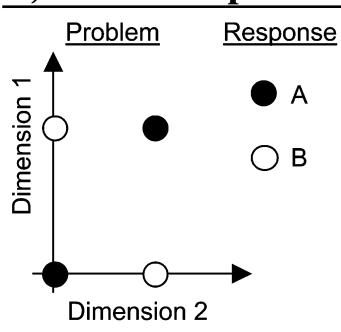

\section{C) Noise $\mathcal{I}$ as a function of $\eta$}

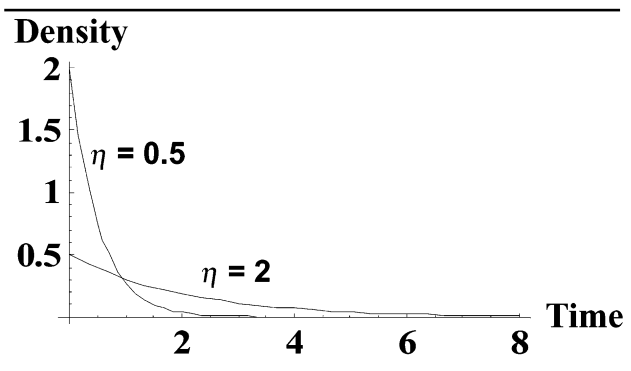

\section{B) Optimal solution}

\begin{tabular}{|c|c|c|}
\hline \multirow{4}{*}{$D=$} & $t_{0}$ & $t_{1}$ \\
\hline & $t_{0}$ & $t_{1}$ \\
\hline & $t_{2}$ & $t_{3}$ \\
\hline & $t_{2}$ & $t_{3}$ \\
\hline$K=$ & $\rho+1$ & 1 \\
\hline
\end{tabular}

D) Noise $\mathcal{D}$

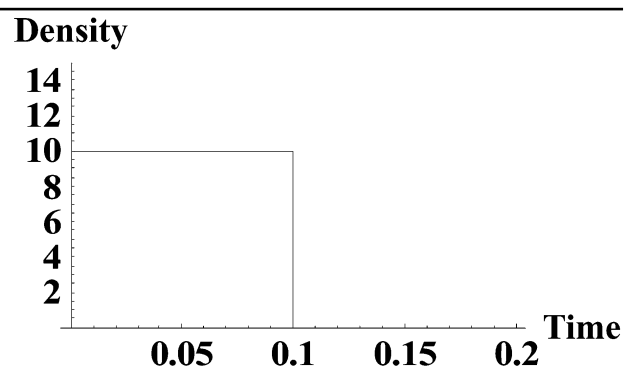

Figure 3. The XOR problem. (A) Response $A$ is correct if both or none of the inputs is on. Response $B$ is made in the case in which only one of the two inputs is on. This is a nonlinearly separable problem. The solution requires two clocks when no input is present. (B) The optimal solution when redundancy is present. (C) Illustration of the distribution of noise added to the input in the simulations. Two levels were tested: $\eta=0.5$ and $\eta=2.0$. (D) Illustration of the distribution of noise added to the delays. 
that the conjunction is present, since both activations could result from the same redundant input. In fact, if one dimension can send $\rho$ redundant activations, only an accumulator of size $\rho+1$ or greater can guarantee that two inputs are present. On the other hand, receiving a single evidence is enough for the alternative decision, B. Yet this decision must not be hasty, since other evidences might be on their way. Thus, the delay between any input and Accumulator B $\left(t_{1}\right)$ should be large, at least larger than the average time to receive $\rho+1$ evidences on Accumulator A.

To check the ability of the PRN to learn this problem, simulations were run in which both $I$ and $D$ were made noisy and redundancy was manipulated. The delay matrix $D$ was made noisy on every trial by adding uniform random delays in the range $[0,0.1]$ in arbitrary units of time $\left(\boldsymbol{D}_{i j}=D_{i j}+\right.$ Uniform $\left.[0,0.1]\right)$. This represents a small amount of noise, but since it is additive with noise in the input, the total amount of noise will be important in some simulations.

The inputs were either 0 , if the dimension was on, or 10 , if it was off (instead of $\infty$ ). This value of 10 means that, after a while, an absent input will nevertheless result in an activation. Indeed, if there is no signal, there is still the possibility that the input unit receives a spurious signal from random activation. Noise was added to the inputs, using an exponential distribution. The magnitude of the noise is described by the mean delay $\eta$ added to the inputs $\left(\boldsymbol{I}_{\boldsymbol{i}}=I_{\boldsymbol{i}}+\right.$ Exponential $\left.[\eta]\right)$. Thus, $\eta$ is also the standard deviation of noise in $\boldsymbol{I}_{i}$. The choice of the uniform and the exponential distributions is arbitrary. Note that on some trials, the noise in one of the two inputs may be quite large (exceeding $\boldsymbol{t}_{1}$ ), so that this information will be too late and a B response will occur. Because the exponential is unbounded, there will always be cases in which diagnostic information will arrive too late.

Figures $3 \mathrm{C}$ and $3 \mathrm{D}$ show the two distributions of noise that were added to the input and to the delay matrix. $\eta=$ 0.5 and $\eta=2.0$ were used for small and large amounts of noise, respectively, in arbitrary units of time. $\eta=0$ means that no noise at all was added to the input.

At the beginning of a simulation, all thresholds $K_{j}$ are set to 1 , and delays $D_{i j}$ are set to random uniform numbers between 0 and 1 in arbitrary units of time. Two clock units were added to all inputs. Training was performed by selecting an input-output pair randomly. For example, $\{0,0\} \rightarrow\{0, \infty\}$ means that when both inputs are on (pattern $\{0,0\})$, Accumulator A should respond first. Noise in $I$ and $D$ was added independently for each input and each channel for each trial. The changes in delay, $\varphi$, were set to 0.5 , and the changes in threshold, $\varepsilon$, were set to 0.2 . The time required to fill each accumulator, $O_{j}$, was obtained by finding the $K_{j}$ th fastest input, and the fastest accumulator made a response. An epoch of training consisted of 10 trials. The network was trained for 200 epochs.

In the second set of simulations, redundancy $\rho$ was manipulated by providing $\rho$ paths where information on
Dimensions 1 and 2 and the two clocks could travel (for a total of $4 \rho$ inputs). Noise was added independently to each input. Accordingly, the rows in the matrix $\boldsymbol{D}$ were duplicated, so that each input $i$ had its own connection to the accumulators.

\section{Results of the Simulations}

The learning capabilities of the PRN are first presented in the presence of noise and then with redundancy. Figure 4 shows the proportion of errors [P(e)] made by the network averaged over epochs. In Figure 4, there is no redundancy $(\rho=1)$. As can be seen in panel A, learning the XOR problem is very rapid (110 trials) when no noise is added to the input $(\eta=0.0)$. Such fast learning is surprising, since strength-based networks typically require hundreds or thousands of trials to do the same (O'Reilly, 1996). The right part of the figure shows the solution adopted by the network (compare with the matrix solution in Figure 3B). $\boldsymbol{t}_{2}$ is represented by the largest delays (8.04 arbitrary units of time). $\boldsymbol{t}_{0}$ corresponds to an average base delay of 4.05 . The increase in $\boldsymbol{t}_{0}$ occurred while the thresholds were stabilizing at the correct values. It took about 100 trials to adjust them.

Figures $4 \mathrm{~B}$ and $4 \mathrm{C}$ show two learning sequences for the XOR problem with two levels of noise in the signal ( $\eta=0.5$ and $\eta=2.0)$ but, again, no redundancy $(\rho=1)$. As can be seen, after 2,000 trials, the network still produced a few errors, but they were well spread out and resulted only from noise. Four patterns of error are in fact possible. On a $\{0,0\}$ input, one of the channels may be slow to be activated, resulting in a false $\mathrm{B}$ response. On a $\{0, \infty\}$ or $\{\infty, 0\}$ input, the activated dimension could be slow, or else spurious activation could occur early on the inactivated channel, resulting in false A responses. Finally, when nothing is presented (pattern $\{\infty, \infty\}$ ), spurious activation could happen earlier than the clocks, resulting in a false $\mathrm{B}$ response. Remember that $\infty$ was replaced by 10. As the number of epochs is increased, errors become more and more sparse. Because this network has no tolerance to errors, learning occurs on every error. For $\eta=$ 2.0 , the network produced $2 \%$ of errors in the last 100 epochs. To avoid errors, the system adapted to the variability in the input by increasing the average delay between $\boldsymbol{t}_{0}$ and $\boldsymbol{t}_{1}$ and between $\boldsymbol{t}_{1}$ and $\boldsymbol{t}_{2}$, as can be seen in the right of the figure.

Apart from noise, redundancy $\rho$ was also manipulated. When $\rho$ is larger than 1, the input can access the system by more than one path. The following set of simulations tried to assess the impact of redundancy on learning.

Figure 5 shows the impact of redundancy $(\rho=1,2,4$, 8 , and 16) for two levels of noise $(\eta=0.5$ and 2.0). As can be seen, learning is slightly slowed down by the redundant sources of incoming signals, errors reaching a lower asymptote in about 1,500 trials for $\rho=16$. This means that the extra inputs learn rapidly to coordinate their action (through the delays $D_{i j}$ ). Furthermore, adding 
$\eta=\mathbf{0 . 0}$
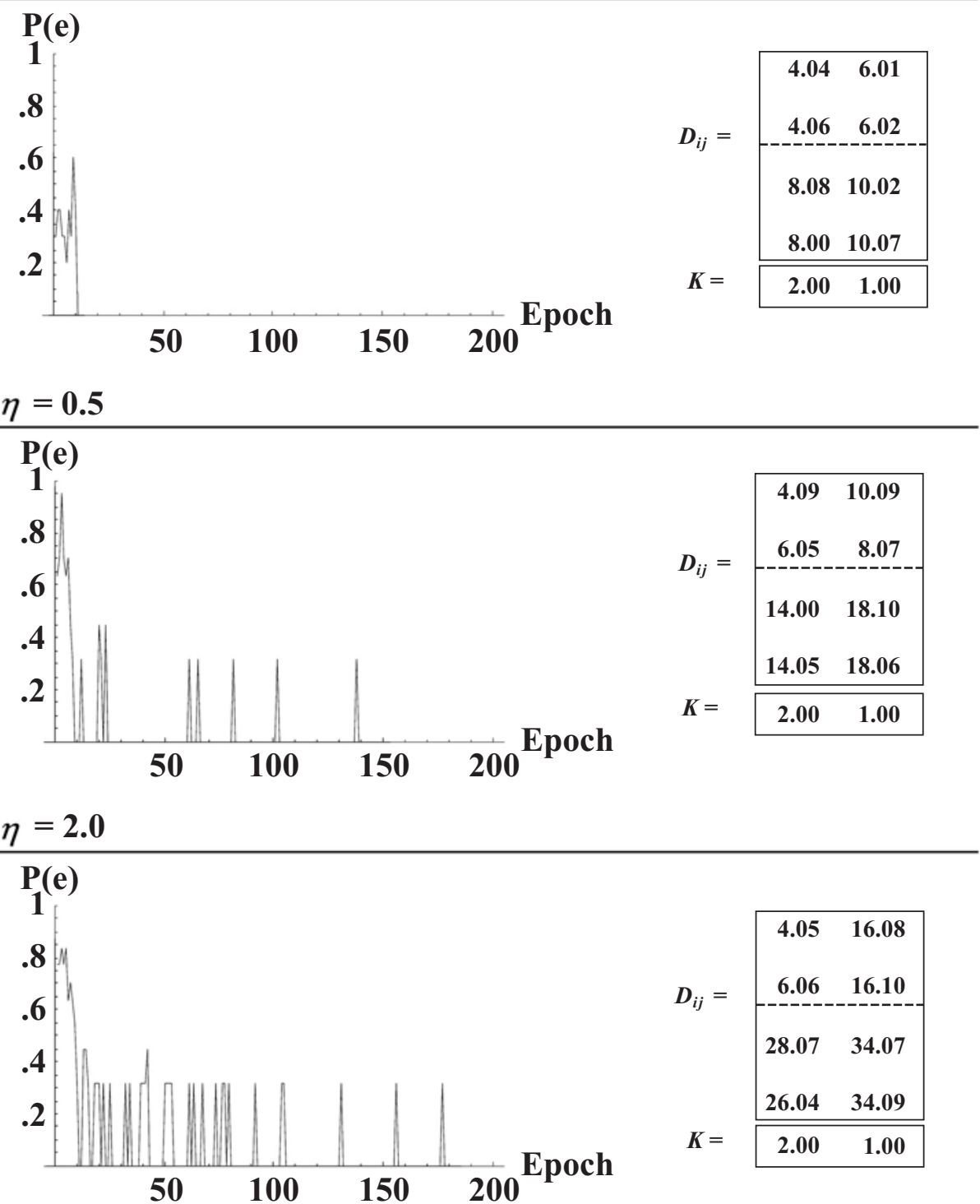

Figure 4. Learning curves of the parallel race network facing the XOR problem. The left panels show the proportion of errors $[P(e)]$ as a function of epoch (one epoch represents 10 trials) when the amount of noise $\eta$ varies from 0.0 to 2.0 ( $\rho$ was constant at 1 ). As can be seen, learning is fast, but in the presence of noise, the system continues to produce scattered errors. The right panels show the solutions obtained by the network. As can be seen, delays are increased when noise increases.

noise in conjunction with redundancy did not hinder learning, the network always finding a stable configuration with only occasional errors.

In sum, the PRN can learn a difficult problem very rapidly, even in the presence of noise and redundancy.

\section{NOISE AND THE PRN}

As was seen in the previous section, the PRN can learn in the presence of noisy input and noisy connections. It can also learn when the difficulty of the problem is multiplied by the presence of redundancy. For strength-based networks, it is known that the addition of a small amount of noise in the connection weights prior to learning helps in reaching a solution (Izui \& Pentland, 1990). However, the impact of constantly adding noise to the weights on every trial, as well as the impact of noise on the stimuli themselves, is not documented. Preliminary work by Cousineau suggested that the strength-based networks rapidly saturate and can no longer learn.

In the previous simulations, noise occurred at two different locations: (1) in the signal incoming time $\boldsymbol{I}$ and 

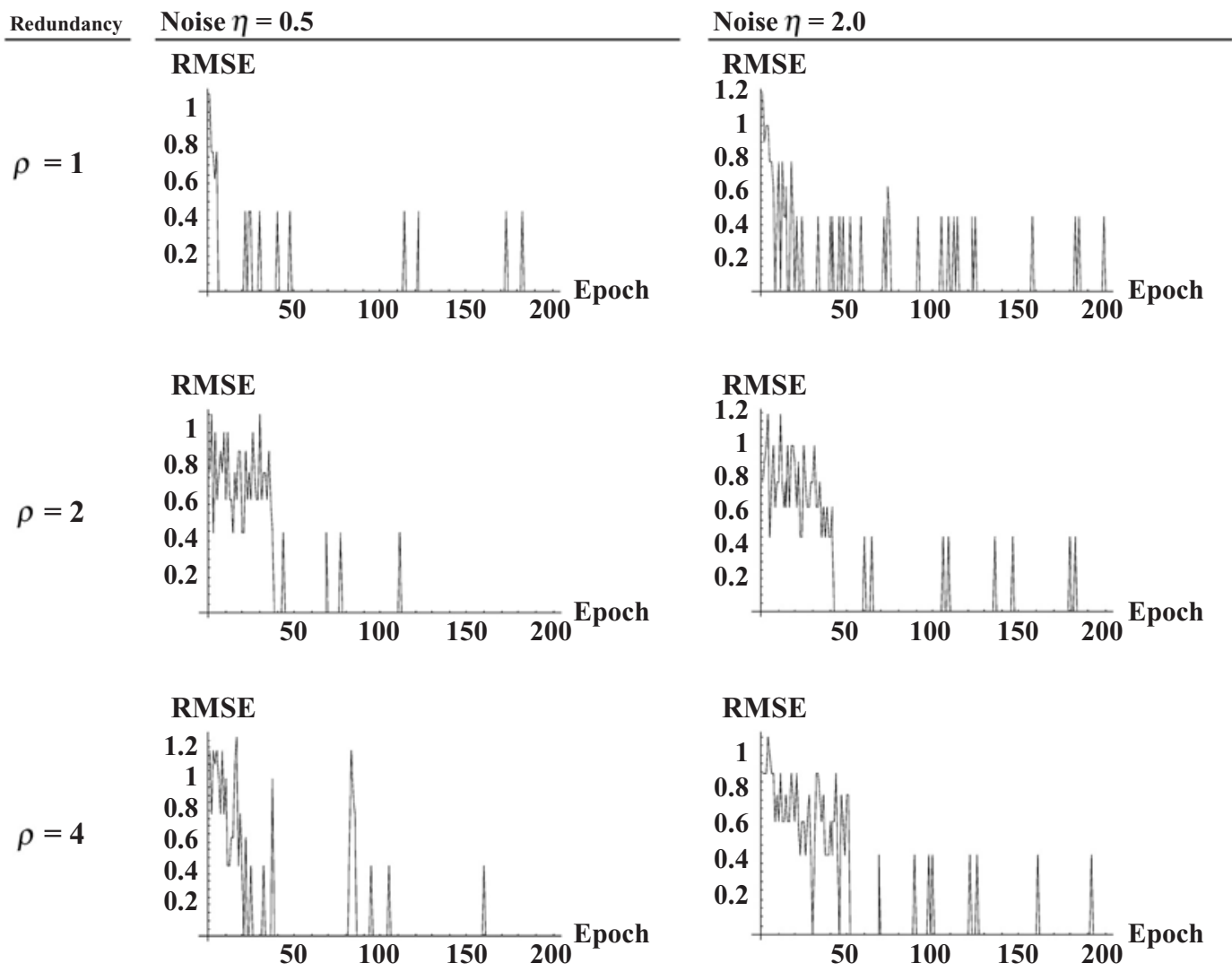

\section{RMSE}

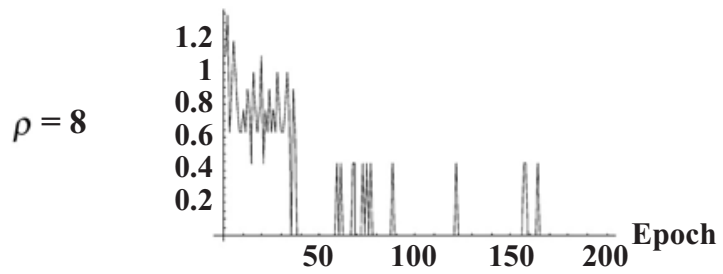

RMSE
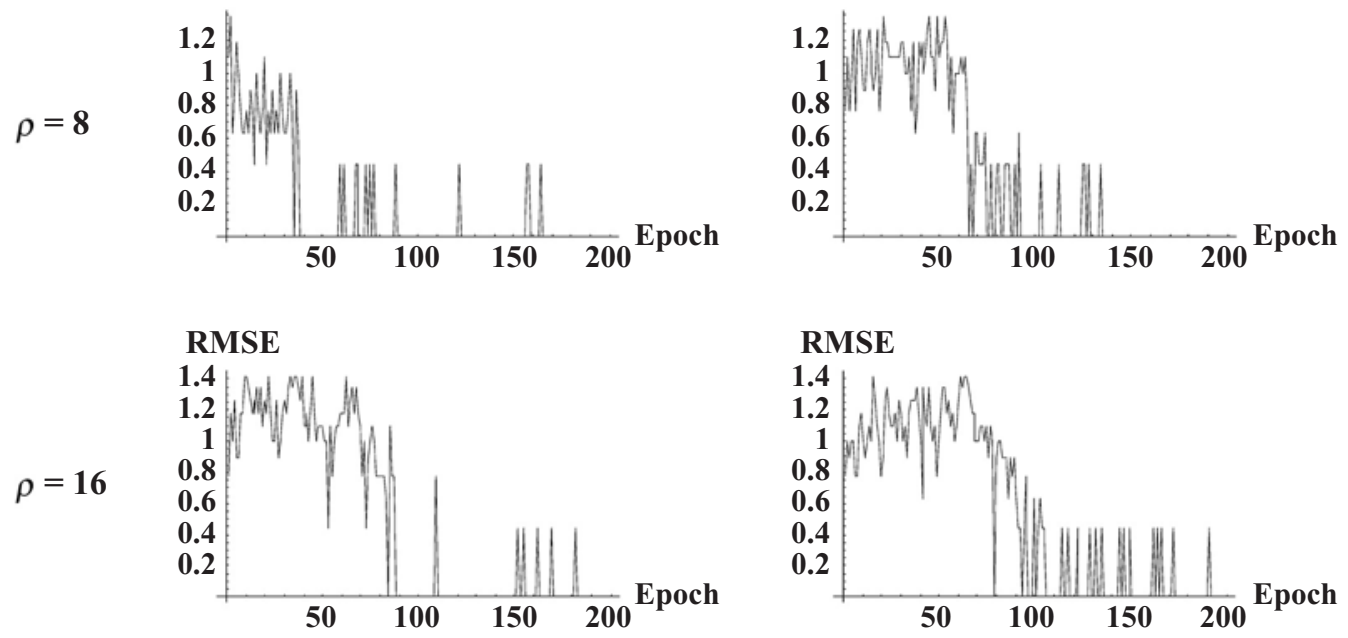

Figure 5. Learning curves of the parallel race network facing the XOR problem when both redundancy $\rho$ and noise $\eta$ are manipulated. Columns show two levels of noise $(\eta=0.5$ and 2.0$)$; rows show five levels of redundancy $(\rho=1,2,4,8$, and 16). Even with large amounts of redundancy and noise, learning occurs rapidly.

(2) in the delays $\boldsymbol{D}$. Randomness in $\boldsymbol{I}$ can be seen as the result of imperfect transmission of information. For example, if information is an impulse traveling on a neuron membrane, the distance traveled or impurities in its composition can introduce small variations in the transmission rate. Delays in $\boldsymbol{D}$ could be variable because of the availability of chemical neurotransmitters and their release, which requires a certain amount of energy.

In the presence of noise, response accuracy can be difficult to achieve. If two distinct responses rely on common inputs, they might fire at approximately the same moment. Any small difference in the transmission times 
resulting from noise might determine which response is first, irrespective of the correct response. ${ }^{3}$ To avoid this, one of the two responses has to be delayed. Thus, a parallel race network must make sure that a fast response for one alternative is accompanied by a slow response for the other alternative. Hence, the presence of noise changes nothing with respect to how the network learns. It is always a question of delaying less diagnostic information and waiting for a critical number of relevant activations. Noise, introducing a larger uncertainty for how long to wait, simply increases the delays: The alternative response waits longer if the diagnostic inputs are often delayed. This is a natural extension of the underlying assumptions of the PRN.

To illustrate this point, we looked at the response times given by the PRN in a previous simulation in which $\eta=$ 0.5 and $\rho=1$ after 60 trials of training. The solution reached by the network at that point was very similar to the one presented in the top part of Figure 4. According to this solution, the stimulus where both inputs are present $(\{0,0\})$ should trigger the first response at time $t=$ 4.06. This, however, disregards the fact that there is noise altering the stimulus and the connection delays. The response will be made, at best, at that moment, resulting in a distribution of activation times for the first accumulator $\boldsymbol{O}_{\mathrm{A}}$.

The first row of Figure 6 shows the distribution of activation times produced by each of the two alternatives when the stimulus $\{0,0\}$ was presented. To obtain these results, the learning process was suspended at trial 60 , a point at which the network was still producing errors. Each stimulus was then presented 5,000 times to the network, and the time to fill each accumulator was recorded. As can be seen from Figure 6, top row, the first accumulator generally fired after 4-7 units of time. The other accumulator did so after 6-7 units of time. The third and fourth columns show the activation time distributions conditional on the fact that the accumulator won the race (i.e., was faster than the other accumulator). In the case of the stimulus $\{0,0\}$ (top row), Response $A$ is the correct response, so that whenever $\boldsymbol{O}_{\mathrm{A}}<\boldsymbol{O}_{\mathrm{B}}$ on a given trial, the network is correct. As can be seen, the network is correct in over $98 \%$ of the trials, even though the overlap between the activation time distributions $\boldsymbol{O}_{\mathrm{A}}$ and $\boldsymbol{O}_{\mathrm{B}}$ is $4 \%$. Similar degrees of overlap are seen for the other three stimuli.

This, incidentally, illustrates one difference between models with a constant decision criterion (such as SDT; Green \& Swets, 1966) and race models. In SDT, the probability that activation strength is above a certain constant $c$ is exactly the probability of a correct rejection (if no signal is presented) or a miss (if a signal is presented). In a race model with two alternatives, the activation time for the first accumulator must be below the activation time of the second accumulator for the first response to occur. Thus, the second accumulator activation time can be seen as a criterion that fluctuates randomly from trial to trial. If the two quantities $\boldsymbol{O}_{\mathrm{A}}$ and $\boldsymbol{O}_{\mathrm{B}}$ were independent, the overlap between their respective distributions would rep- resent exactly the percentage of errors, but because they are partly dependent (they are generated from the same input), the percentage of error is smaller than the degree of overlap, as is the case in Figure 6.

Inspection of Figure 6 indicates that the variability (the width of the distributions) is the same for all the stimuli. This is true because, in the simulations, the amount of noise did not depend on the pattern presented. The width of the distributions thus is proportional to $\eta$. On the other hand, the position of the distributions, the point at which they start to rise, depends on the stimulus presented and the accumulator considered. The positions are 4 and 6 for stimulus $\{0,0\}, 8$ and 6 for stimuli $\{0, \infty\}$ or $\{\infty, 0\}$, and finally, 8 and 10 for stimulus $\{\infty, \infty\}$. They correspond to the base response times found in the delay matrix $D$. They are seen in the solution given in Figure 4 (they were referred to as $\boldsymbol{t}_{0}, \boldsymbol{t}_{1}$, and $\boldsymbol{t}_{2}$ in Figure 3 ). The network learned these positions. They were increased to reflect the order of priority of the responses.

In sum, the position parameters can be used to reconstruct one component learned by the network, the matrix $D .{ }^{4}$ However, as the following shows, the second component of the network, the accumulator sizes $K_{j}$, cannot be identified from distribution analyses. We will first examine how to derive the distribution of response times for each accumulator and the distribution of correct and error responses, and then we will examine the distribution parameters and how they relate to the components of the PRN.

When $I$ and $D$ are deterministic (not random) variables, the solutions $\left(\boldsymbol{t}_{0}, \boldsymbol{t}_{1}, \boldsymbol{t}_{2}\right)$ are the exact activation times of the accumulators, and so the $O_{j}$ s are also deterministic. However, with random input and delays, the type of distribution of activation times $\boldsymbol{O}_{j}$ has to be inferred using statistical arguments. As was done in the simulations, we add noise in two places:

$$
\boldsymbol{I} \leftarrow I+\text { noise }
$$

and

$$
\boldsymbol{D} \leftarrow D+\text { noise },
$$

and the amount of noise can be summarized by the variance or, more simply, by the standard deviation in the delays ( $\eta$ in the simulations).

The noise in $\boldsymbol{I}$ and $\boldsymbol{D}$ must be characterized by some distribution functions - say, $\boldsymbol{I}$ and $\mathcal{D}$ - which must be meaningful in the time domain. The most important restriction is that they never assume negative values. Indeed, noise cannot make a signal available earlier. As such, the Gaussian distribution (among others) should not be used in conjunction with the PRN.

The first step is to infer the distribution of times before the $j$ th accumulator is filled. Call this distribution $\boldsymbol{O}_{j}$, which is a function of the input and the delay matrix $I$ and $\mathcal{D}$. To solve analytically the distribution function of the $j$ th accumulator, redundancy is assumed to be large, so that asymptotic theories can be used. The problem is to find the distribution of the $K_{j}$ th fastest incoming signals from a large pool of signals $\left(K_{j}\right.$ small relative 
Frequency of response times
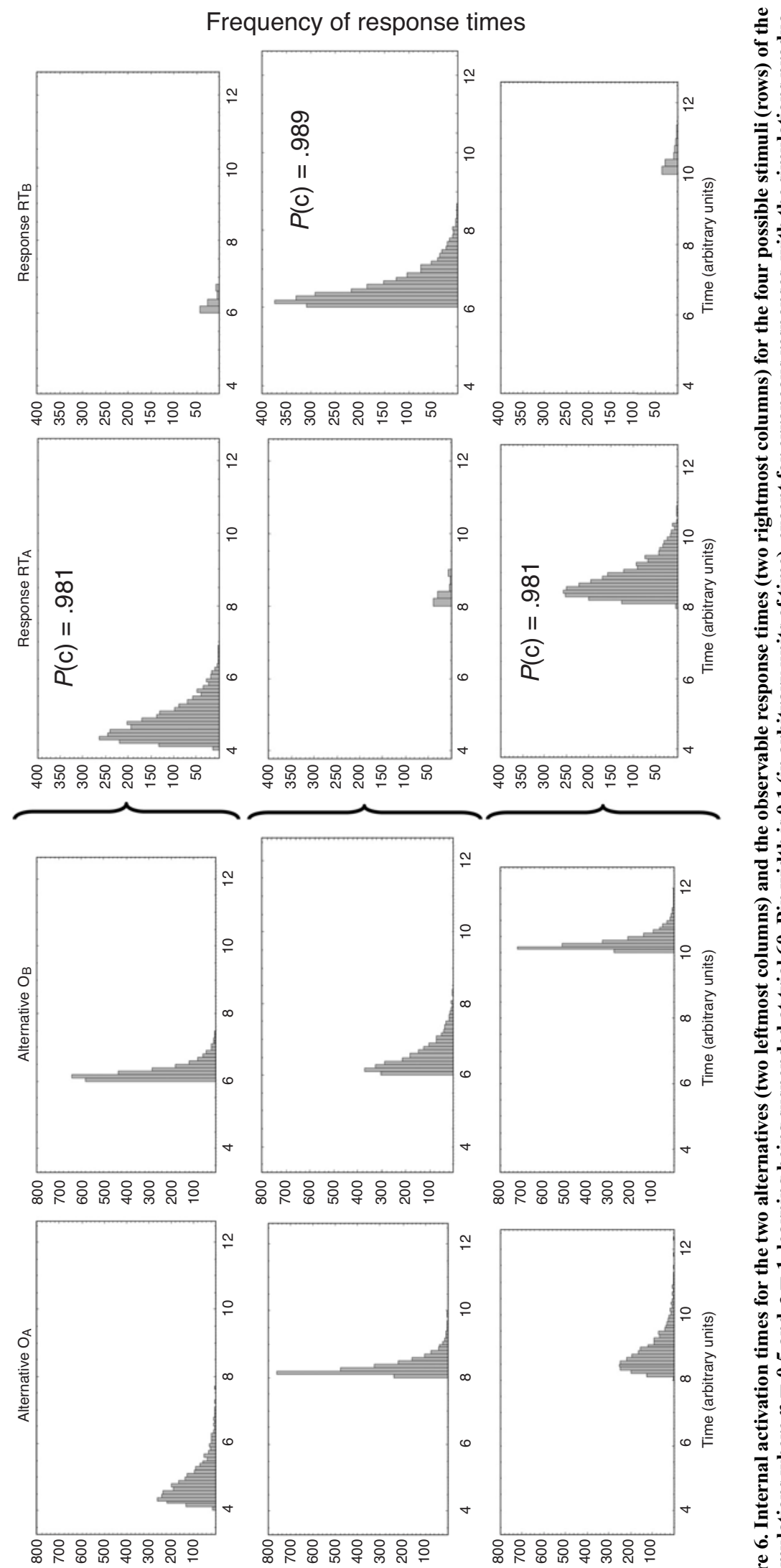

\{0'0\} sn!nu!!s

$\left\{0^{\prime} \infty\right\}$ до $\left\{\infty^{\prime} 0\right\}$ !num!̣s
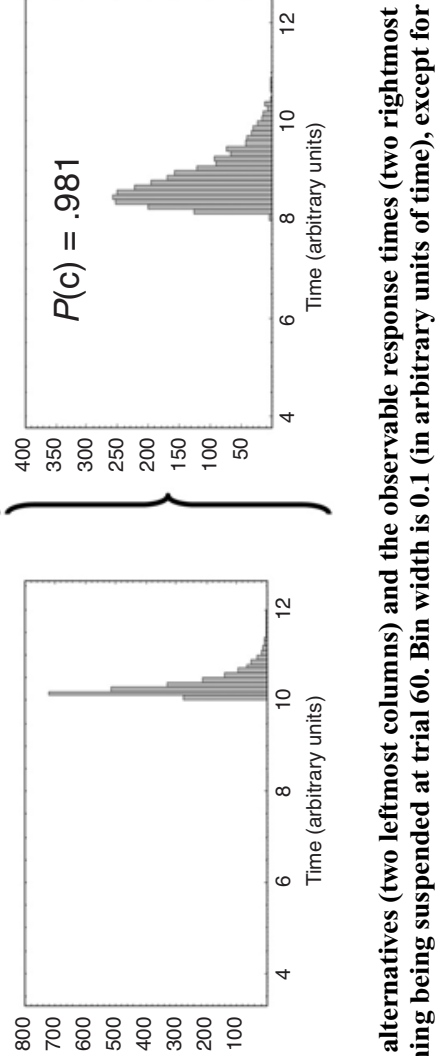

踏
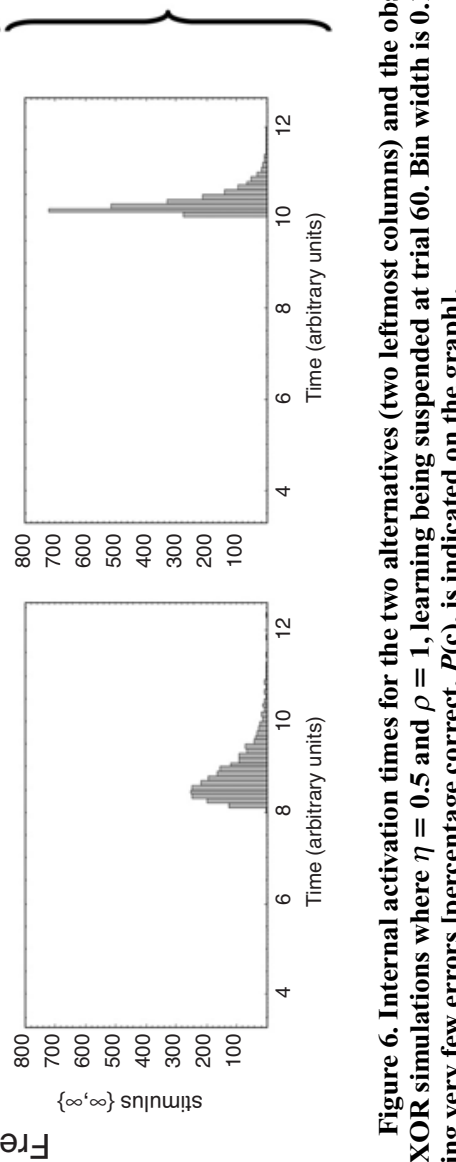
to the pool size). Statistics of extremes (minima or maxima) have been used to study such problems (Cousineau, Goodman, $\boldsymbol{\&}$ Shiffrin, 2002). We can write $\boldsymbol{O}_{j}$ as a distribution function given by

$$
\boldsymbol{O}_{j}=L_{\sim}(\boldsymbol{I}, \mathcal{D}),
$$

where $L$ returns the asymptotic (i.e., redundancy large) distribution of $\boldsymbol{I}$ and $\mathcal{D}$ with respect to the operator $\sim$. Now, because the first step is to join - that is, to sum - the delays in the input and the delay matrix, a convolution describes the combination of $\boldsymbol{I}$ and $\mathcal{D}$ (Cramér, 1946; Feller, 1957), and we can note that

$$
\boldsymbol{O}_{j}=L_{\operatorname{Min}_{K}}(\boldsymbol{I} * \mathcal{D})
$$

Thus, solving the accumulator's distribution is simply to find the asymptotic distribution with respect to minima, a well-known problem in the statistics of extremes (Galambos, 1978; Gumbel, 1958), yielding Weibull distributions for the accumulators:

$$
\boldsymbol{O}_{j}(t)=W_{\alpha, \beta, \gamma}(t)
$$

where $W$ is the Weibull distribution whose cumulative and density functions are given by

$$
F(t)=1-e^{-\left(\frac{x-\alpha}{\beta}\right)^{\gamma}}
$$

and

$$
f(t)=\beta^{-\gamma} \gamma(t-\alpha)^{(\gamma-1)} e^{-\frac{(t-\alpha)^{\gamma}}{\beta}},
$$

respectively (Cousineau \& Larochelle, 1997; Luce, 1986; Weibull, 1951). ${ }^{5}$ In these equations, $\alpha$ is the position parameter (also called a shift parameter; Cousineau, Brown, $\&$ Heathcote, 2004), $\beta$ is the scale parameter (akin to standard deviation), and $\gamma$ is the shape parameter (akin to skew). In sum, if redundancy is high, the activation time of each accumulator ought to be distributed as a Weibull.

A second step is needed because the $\boldsymbol{O}_{j}$ s are not necessarily observable. Because only the fastest accumulator produces a response, the observed distributions are distorted in some predictable way by the other responses' distributions. Let $\mathscr{R} \mathcal{T}_{j}$ be the observed distributions of response times for the response $j=\mathrm{A}, \mathrm{B} \ldots$ It is given by the probability that the accumulator $j$ fires at time $t$ multiplied by the probability that the other accumulators finish later, denoted

$$
\mathcal{R T}_{j}(t)=\frac{1}{P_{j}} f_{j}(t) \prod_{k \neq j}\left[1-F_{k}(t)\right]
$$

where

$$
P_{j}=\int_{0}^{\infty} f_{j}(u) \prod_{k \neq j}\left[1-F_{k}(u)\right] d u
$$

is a normalizing value to make sure the density function has an area of 1 (Huber \& Cousineau, 2003; Van Zandt, Colonius, \& Proctor, 2000). Thus, the observable $\mathfrak{R} \mathcal{T}$ distributions are not Weibulls; in fact, the equation above does not have a closed form solution in most situations.
Nonetheless, it can be fit to data (Huber \& Cousineau, 2003; Van Zandt, 2000), using maximum likelihood methods (Cousineau et al., 2004), so that the best-fitting parameters $\alpha, \beta$, and $\gamma$ for each accumulator are estimated.

Given the equations that describe the observable behavior of the PRN, it remains to be seen how the resulting parameters relate to the state of the network (i.e., the learned delays $D$ and accumulator sizes $K$ ) and the characteristics of the noise present throughout the system $(\eta$ and $\rho$ ). As was seen at the beginning of this section, the position parameter $\alpha$ indicates the base delays in the $D$ matrix. The scale parameter $\beta$ estimates the amount of noise at the output and is a function of both the noise at the input and in the delays and the amount of redundancy. As can be seen in statistics of extreme (e.g., Cousineau et al., 2002), the more redundant the racers are, the less noisy the winner is. The general relation between $\eta, \rho$, and $\beta$ is of the form $\beta \propto \eta / \sqrt{ } \rho$. Therefore, having an estimate of $\beta$, it is not possible to identify both $\eta$ and $\rho$. Thus, the amount of internal redundancy, if such a thing is present, cannot be estimated with this type of fitting. Using a measure of capacity developed by Townsend (e.g., Townsend \& Nozawa, 1995), Cousineau (2001) proposed an alternative approach for isolating $\rho$ that awaits an empirical test. Finally, $\gamma$ is a signature of the family of noise present in the system. As such, it should be a constant. Huber and Cousineau (2003) used a fixed $\gamma$ parameter (at 1.3) for all the subjects and all the conditions, without a significant decrement in fit. In the previous simulations, with a combination of exponential and uniform noise, $\gamma$ is predicted to be 2 , as indicated by Theorem 1 of Cousineau et al., 2003.

To recapitulate, from distributional analyses, we can extract information given by the parameters $\alpha, \beta$, and $\gamma$ from each stimulus and each accumulator. The position parameters $\alpha$ allow estimation of the delay matrix $D$. The scale parameter aggregates both $\eta$ and $\rho$. Finally, $\gamma$ is a signature of the family of noise present in the system. As can be seen, there is a loss of information: From the observable distributions, no parameter relates in any way to the thresholds $K_{j}$. Alternative approaches might have more success (see, e.g., Meyer, Irwin, Osman, \& Kounios, 1988). Overall, the PRN reduces the errors by increasing the delays of less diagnostic information. If noise is present, this results in a decrease of the overlap between accumulators' response times. This is seen by a shift in distribution that should be easy to identify through an experiment with human subjects. This issue is being explored in current works.

\section{GENERAL DISCUSSION}

The PRN is a race model because of the competition between accumulators. However, it is also a connectionist network by its architecture and its ability to learn (McClelland \& Rumelhart, 1988). Yet its learning ability is not limited by linearly separable problems (Minsky \& Papert, 1969). Indeed, the PRN learned to classify rapidly all the two-dimensional problems considered in this arti- 
cle, with or without noise and with or without redundancy. This model is tailored to predict response times, because the notion of time is the primitive of this system. Furthermore, assuming large redundancy, the extreme limit theorem can be used to solve the model analytically. Cousineau et al. (2002) showed that when the aggregate operator is a minimum (as is the case for $\sim$ ), the distribution of the $K$ th minimum is a Weibull distribution.

Because the network needs to know that time is going on when nothing else is happening, clock units were added to the system. Although they add dimensions to the inputs (not related to the inputs, since the clocks are always on), they do not make the problems harder to solve. On the contrary, because they represent chronometers that tick the seconds away, they provide positive information that can be used in such situations as "If by now nothing else has happened, you might consider this." Thus, this system learns to prioritize its decision on the basis of the received evidence, and one of its options is "let's wait a little more."

It is tempting to call the PRN a race-race model. Indeed, it is a race model at two distinct levels. First, and following the usual meaning of the expression race model, this model has accumulators, and each one is racing to be the first to trigger a response. At this level, this is a between-accumulator race model. Second, when a single accumulator is considered, there are redundant channels in competition to be the first to fill the accumulator. Thus, there is a race going on within each accumulator.

More important, however, is the fact that the PRN opens new research ideas and avenues of inquiry. For example, it shows how supervised connectionist networks and accumulator models can be related and unified under a unique network architecture. The fundamental difference between the two is the use of summation or minimum to evaluate the incoming evidence. In the first case, the evidences are meaningful if thought of as strength of the input and strength of connections, whereas in the second case, the evidences are seen in the time domain as moments at which the inputs become available and what priority they must receive with delays in the connections. Finally, the PRN motivates new methods for computing a network's output. Indeed, redefining the inner product opens a totally new area for matrix operations.

\section{Some Properties of the PRN}

The PRN has two properties that are very important for psychological plausibility - namely, reduction of variability and resistance to partial destruction.

The reduction of variability is a useful property for any psychological model. Suppose that the units have a slow conductance rate and a significant amount of variability resulting from noise $\eta$ (as is the case for neurons, for example). A serial system composed of $m$ stages would end up with a tremendous amount of variability $m \times \eta$. In parallel systems, adding a second unit in parallel, whose purpose is the same as the first one, and considering only the fastest, variability decreases considerably. In some cases, with only two redundant units $(\rho=2)$, variability of the output is reduced to half the variability of the incoming signal, with three, it is reduced to one third, and so forth. In general, the resulting amount of noise from $\rho$ redundant inputs is proportional to $\eta / \sqrt{2} \rho$, where $\gamma$ depends on the shape of the variability of each unit (Cousineau et al., 2002). This provides an easy way to reduce variability (noise). No special filters or transformations are needed. In fact, it is possible to construct a serial system composed of $m$ layers, where each layer is composed of units racing in parallel, with variability at the output that is comparable in magnitude with the variability of the inputs. ${ }^{6}$ Such a system is said to be in control of the noise and can be extended to a larger number of layers without much decrease in performance resulting from noise.

Resistance to partial destruction also results from the assumption of redundancy: With a large number of redundant channels, loss of a few will barely affect the system. Perhaps the only visible manifestation of partial destruction would be a small rise in variability, since fewer units are now competing. These two properties do not depend on the nature of the units (neurons or others). They are emergent properties of large collections of random elements, where only the fastest are crucial at any given moment.

\section{Reduction of Information and Serial Effects}

The PRN exhibits two more properties that could be relevant to human cognition. First, because this model is expected to react as soon as possible, it is assumed that the accumulator sizes are the shortest possible (given constraints on accuracy). The learning rule presented in the A PRN With a Learning Rule section also tends to prefer shorter, rather than longer, accumulators. This model, therefore, disregards unneeded information, which makes it compatible with reduction-of-information theories. Haider and Frensch (1996, 1999; Cousineau \& Larochelle, in press) postulated such a model, in which only the smallest subset of diagnostic information is used to base a decision. Thus, parallel race models could be used to implement a reduction-of-information theory.

Second, from a neuroanatomical point of view, the weight of evidence clearly suggests that the brain has a massive parallel architecture. Yet in cognitive psychology, there are countless box-and-arrow models in which certain operations must be performed in a given sequence. Some of these models implicitly assume a central executive that knows when to perform the operation. This only relegates the notion of time one notch deeper (van Gelder, 1995). One elegant aspect of the PRN is that it does exactly the opposite: Time is the front end of this parallel architecture. The PRN responds more quickly to more complex patterns, since it relies on positive evidence. Because the absence of information has no representation in this network, the various accumulators in competition must agree to defer their response a little longer when they might be in the presence of slow inputs.

This results in the most interesting property of all: apparent seriality out of a parallel architecture. The serial 
effects obtained cannot be schematized appropriately by a box-and-arrow diagram, since the $B$ response will occur if Response A was not triggered, not after Response A was discarded. The distinction is subtle but very important, since it implies that Response B has no real knowledge whether processing for an A response was completed or not. This fake seriality may pose problems for those who analyze their data in the context of the additive factor method (Sternberg, 1969) and the descendant of this method, the mean interaction contrast (Thomas, 2000; Townsend \& Nozawa, 1995). Further studies are needed to see whether statistics such as the mean interaction contrast and properties such as the Miller (1982) inequality still hold in the parallel race model (Cousineau, 2001; Ulrich \& Giray, 1986). Nevertheless, this model provides a convenient framework, which is, at the same time, intuitive and highly constrained by mathematical relations.

At this point, the PRN has some properties that might be present in human information-processing capabilities. However, others are not. For example, there is no carryover of information from one trial to the other, since the accumulators are reset between trials. As such, the PRN could not explain priming phenomena. This could be remedied by postulating that there is no reset but that each accumulator has a strong tendency to return to zero. This could be implemented using leaky integrators (Usher \& McClelland, 2001).

\section{Thorpe's Paradox and the Learning Rule}

In light of the paradoxical quotation from Thorpe and Gautrais (1999), presented in the introduction, an interesting question emerges: If only the first few milliseconds of neural activity is enough to trigger a response, why does the activation last so long? In the PRN, only the moment when the activation turns on is relevant. It could shut down immediately after. Therefore, long activation is not relevant for decision making. But might it be relevant for learning?

In classic supervised networks, learning occurs through a "teacher" that can indicate, for each output unit, how much it was wrong. However, such a detailed feedback is controversial (Page, 2000). Assuming that feedback cannot be that specific, what kind of signal would constitute an acceptable feedback? The simplest feedback might be an imperative signal to stop whatever is currently being executed. Such a stop mechanism could be implemented using other, more primitive parts of the system, such as reflexes (remember the first time you touched a burning stove). The only relevant role of such feedback is to stop immediately what would otherwise be an excessively long period of high activation. Consequently, a long period of high activation might serve as a confirmation: If a behavior is not interrupted quickly, it is not erroneous. Therefore, neuron activities could be characterized using two dimensions: the moment when activity turned on and its duration. These two dimensions are responsible for two distinct behaviors: decision and learning. This view could resolve some of the contro- versy surrounding the notion of "teachers" in supervised learning.

\section{A Final Word}

Ratcliff et al. (1999), who extensively explored RT distributions and phenomena such as SATO, remarked, "The findings ... show that the long tradition of reactiontime research and theory is a fertile domain for development and testing ... how decisions are generated over time" (p. 261). I would like to stress this point even more: Maybe time is the fundamental unit of cognition. At least, I have shown in this article that, in principle, it could be so.

\section{REFERENCES}

Arguin, M., \& Bub, D. (1995). Priming and response selection processes in letter classification and identification tasks. Journal of Experimental Psychology: Human Perception \& Performance, 21, 11991219.

Audley, R. J., \& Pike, A. R. (1965). Some alternative stochastic models of choice. British Journal of Mathematical \& Statistical Psychology, 18, 207-225.

Changeux, J.-P. (1983). L'homme neuronal. Paris: Fayard.

Chaplain, R. A. (1979). Metabolic control of neuronal pacemaker activity and the rhythmic organization of central nervous functions. Journal of Experimental Biology, 81, 113-130.

Cousineau, D. (2001, July). Redundancy conjecture and super-capacity rate of increase. Paper presented at the annual meeting of the Society for Mathematical Psychology, Providence, RI.

Cousineau, D., Brown, S., \& Heathcote, A. (2004). Fitting distributions using maximum likelihood: Methods and packages. Behavior Research Methods, Instruments, \& Computers, 36, 742-756.

Cousineau, D., Goodman, V., \& Shiffrin, R. M. (2002). Extending statistics of extremes to distributions varying on position and scale, and implication for race models. Journal of Mathematical Psychology, 46, 431-454.

Cousineau, D., Lacroix, G. L., \& Hélie, S. (2003). Redefining the rules: Providing race models with a connectionist learning rule. Connection Science, 15, 27-43.

Cousineau, D., \& Larochelle, S. (1997). PASTIS: A program for curve and distribution analyses. Behavior Research Methods, Instruments, \& Computers, 29, 542-548.

Cousineau, D., \& Larochelle, S. (in press). Visual-memory search: An integrative perspective. Psychological Research.

Cramér, H. (1946). Mathematical methods of statistics. Princeton, NJ: Princeton University Press.

Feller, W. (1957). An introduction to probability theory and its application (2nd ed., Vol. 1). New York: Wiley.

Galambos, J. (1978). The asymptotic theory of extreme order statistics. New York: Wiley.

Green, D. M., \& Swets, J. A. (1966). Signal detection theory and psychophysics. New York: Wiley.

GrünWALD, P. (2000). Model selection based on minimum description length. Journal of Mathematical Psychology, 44, 133-152.

Gumbel, E. J. (1958). The statistics of extremes. New York: Columbia University Press.

Haider, H., \& Frensch, P. A. (1996). The role of information reduction in skill acquisition. Cognitive Psychology, 30, 304-337.

Haider, H., \& Frensch, P. A. (1999). Eye-movement during skill acquisition: More evidence for the information-reduction hypothesis. Journal of Experimental Psychology: Learning, Memory, \& Cognition, 25, 172-190.

HintoN, G. E. (1992, September). How neural networks learn from experience. Scientific American, 267, 145-151.

Hopkins, G. W., \& Kristofferson, A. B. (1980). Ultrastable stimulusresponse latencies: Acquisition and stimulus control. Perception \& Psychophysics, 27, 241-250.

Huber, D. E., \& Cousineau, D. (2003, June). A race model of percep- 
tual forced choice reaction time. Proceedings of the 25th Annual Meeting of the Cognitive Science Society (www.ccm.ua.edu/cogsci/).

Izui, Y., \& Pentland, A. (1990). Analysis of neural networks with redundancy. Neural Computation, 2, 226-238.

KoHONEN, T. (1984). Self-organization and associative memory. Berlin: Springer-Verlag.

LABERge, D. A. (1962). A recruitment theory of simple behavior. Psychometrika, 27, 375-396.

LeCun, Y., \& BotTou, L. (2003, June). Lagrangian difference learning. Paper presented at the workshop on advances in machine learning, Montreal.

Levitan, I. B., Harmar, A. J., \& Adams, W. B. (1979). Synaptic and hormonal modulation of a neuronal oscillator: A search for molecular mechanisms. Journal of Experimental Biology, 81, 131-151.

LUCE, R. D. (1986). Response times: Their role in inferring elementary mental organization. New York: Oxford University Press.

McClelland, J. L., \& Rumelhart, D. E. (1988). Explorations in parallel distributed processing: A handbook of models, programs, and exercises. Cambridge, MA: MIT Press.

Meijers, L. M. M., \& EiJKman, E. G. J. (1977). Distributions of simple RT with single and double stimuli. Perception \& Psychophysics, 22, 41-48.

MEYer, D. E., Irwin, D. E., Osman, A. M., \& Kounios, J. (1988). The dynamics of cognition and action: Mental processes inferred from speed-accuracy decomposition. Psychological Review, 95, 183-237.

MiLler, J. (1982). Divided attention: Evidence for coactivation with redundant signals. Cognitive Psychology, 14, 247-279.

Minsky, R., \& PAPERT, S. (1969). Perceptrons: An introduction to computational geometry. Cambridge, MA: MIT Press.

MYUNG, I.-J. (2000). The importance of complexity in model selection. Journal of Mathematical Psychology, 44, 190-204.

O'REILLY, R. C. (1996). Biologically plausible error-driven learning using local activation differences: The generalized recirculation algorithm. Neural Computation, 8, 895-938.

PAGE, M. (2000). Connectionist modelling in psychology: A localist manifesto. Behavioral \& Brain Sciences, 23, 443-512.

PIKe, R. (1973). Response latency models for signal detection. Psychological Review, 80, 53-68.

RATClifF, R., VAN ZANDT, T., \& McKoon, G. (1999). Connectionist and diffusion models of reaction time. Psychological Review, 106, 261-300.

RoSENBLATT, F. (1961). Principles of neurodynamics: Perceptrons and the theory of the brain mechanisms. Washington, DC: Spartan.

Rousseau, L., \& Rousseau, R. (1996). Stop-reaction time and the internal clock. Perception \& Psychophysics, 58, 434-448.

SMITH, P. L., \& VICKERS, D. (1988). The accumulator model of twochoice discrimination. Journal of Mathematical Psychology, 32, 135168 .

STERNBERG, S. (1969). The discovery of processing stages: Extensions of Donders' method. Acta Psychologica, 30, 276-315.

Thomas, R. D. (2000, August). Analysis of factorial response time patterns predicted by current models of perception. Paper presented at the 33rd Annual Meeting of the Society for Mathematical Psychology, Kingston.

ThorPe, S. J., \& Gautrais, J. (1999). Rapid visual processing using spike asynchrony. Toulouse: Centre de Recherche Cerveau \& Cognition.

Townsend, J. T., \& Ashby, F. G. (1983). Stochastic modeling of elementary psychological processes. Cambridge: Cambridge University Press.

Townsend, J. T., \& Nozawa, G. (1995). Spatio-temporal properties of elementary perception: An investigation of parallel, serial, and coactive theories. Journal of Mathematical Psychology, 39, 321-359.

ULRICH, R., \& GIRAY, M. (1986). Separate-activation models with variable base times: Testability and checking of cross-channel dependency. Perception \& Psychophysics, 39, 248-254.

Usher, M., \& MCClelland, M. L. (2001). On the time course of perceptual choice: The leaky competing accumulator model. Psychological Review, 108, 550-592.

VAN GELDER, T. (1995). What might cognition be, if not computation? Journal of Philosophy, 92, 345-381.
VAN ZANDT, T. (2000). How to fit a response time distribution. Psychonomic Bulletin \& Review, 7, 424-465.

VAN ZANDT, T., COlONIUS, H., \& PrOCTOR, R. W. (2000). A comparison of two response time models applied to perceptual matching. Psychonomic Bulletin \& Review, 7, 208-256.

WARD, R., \& MCCLELlAND, J. L. (1989). Conjunctive search for one and two identical targets. Journal of Experimental Psychology: Human Perception \& Performance, 15, 664-672.

WEIBULL, W. (1951). A statistical distribution function of wide applicability. Journal of Applied Mechanics, 18, 292-297.

Widrow, B., \& HoFF, M. E. (1960). Adaptive switching circuits. Institute of Radio Engineers, Western Electronic Show and Convention: Convention Record, 4, 96-194.

\section{NOTES}

1. Because the weights in a connectionist network are partly determined by training, they might be called partly free parameters. To test distinct models, it is often necessary to know the number of free parameters (using, for example, the likelihood ratio test; Grünwald, 2000; Myung, 2000). At this time, no one knows how many partially free parameters it takes to correspond to one fully free parameter. This question is open to further research.

2. A promising approach might be related to minimum descriptive length theory (Grünwald, 2000). Indeed, for a network with $n_{i}$ inputs and, therefore, at most $n_{i}$ useful clocks, the PRN can implement $2 \times n_{i}$ simple rules (either logical rules or counting rules). However, $2^{\text {ni }}$ inputs exist. Therefore, whether the PRN can learn them is a question of whether the inputs can be compressed into $2 \times n_{i}$ rules.

3. A situation in which responding is difficult for the PRN is the case of embedded patterns. For example, consider the and problem in the Deterministic Solutions to Simple Problems section. One pattern of input triggering the first response, call it $I^{\mathrm{A}}$, is $\{0,0\}$, and a pattern that triggers the second response, $\boldsymbol{I}^{\mathrm{B}}$, is $\{0, \infty\}$, where 0 denotes an input activated by the stimulus and $\infty$ an absence of input. The activated unit of $\boldsymbol{I}^{\mathrm{B}}$ is also part of pattern $\boldsymbol{I}^{\mathrm{A}}$. When presented with pattern $\boldsymbol{I}^{\mathrm{B}}$, in order to be sure that $B$ is the right response, the system must be certain that the second channel, $\boldsymbol{I}_{2}$, will not become activated in the next few moments. Yet in the presence of noise, it is impossible to have a perfect confidence that $\boldsymbol{I}_{i}$ is not about to fire. Indeed, suppose that $\boldsymbol{I}^{\mathrm{A}}$ is the pattern presented, but because of noise, the second channel will register the presence of the second attribute at time 50 (in arbitrary units of time). If the delay $D_{1 \mathrm{~B}}$ is smaller than 50 (say, 40), then at time 40, Accumulator $\mathrm{B}$ will be filled by the first evidence, and a response will be made (because accumulator size is 1). This is a false alarm for Response B. One way to keep the false alarm rate low is not to make hasty decisions. The delays should reflect the temporal odds of a particular input in the presence of noise. For example, if $\boldsymbol{I}_{2}$ generally becomes active after 30 $\pm 10 \mathrm{msec}$, given that pattern $\boldsymbol{I}^{\mathrm{A}}$ was presented, then after $60 \mathrm{msec}$ (three standard deviations above 30 ), the system can decide confidently that $I_{2}$ is not present. Therefore, the first input should be delayed at least $60 \mathrm{msec}$ before reaching Accumulator B $\left(\boldsymbol{D}_{1 \mathrm{~B}}=60 \mathrm{msec}\right)$.

4. This assumes that there is no contribution to response time from other processes, such as encoding times or motor times.

5. As can be seen, the result is not based on a specific distribution function. Many noise functions predict a Weibull distribution. As such, to be consistent with the "serial Poisson race model" label (Townsend \& Ashby, 1983), we could call the PRN the "parallel anything race model."

6 . The question here is to find a solution to

$$
\eta_{m} \approx m\left(\frac{\eta_{0}}{\sqrt[\gamma]{\rho}}\right)^{\stackrel{?}{\leq}} \eta_{0},
$$

where $\eta_{m}$ is the noise at the output of the $m$ th stage. In the case in which $\gamma=2$, one obvious solution is $\rho \geq m^{2}$. Therefore, with a system whose "width" is larger than its "depth" squared, such a race model has a stable amount of variability at the output.

(Manuscript received February 27, 2001; revision accepted for publication October 17, 2003.) 